TRANSACTIONS OF THE

AMERICAN MATHEMATICAL SOCIETY

Volume 361, Number 8, August 2009, Pages 4437-4470

S 0002-9947(09)04683-2

Article electronically published on March 9, 2009

\title{
TOWARDS A COMBINATORIAL CLASSIFICATION OF SKEW SCHUR FUNCTIONS
}

\author{
PETER R. W. MCNAMARA AND STEPHANIE VAN WILLIGENBURG
}

\begin{abstract}
We present a single operation for constructing skew diagrams whose corresponding skew Schur functions are equal. This combinatorial operation naturally generalises and unifies all results of this type to date. Moreover, our operation suggests a closely related condition that we conjecture is necessary and sufficient for skew diagrams to yield equal skew Schur functions.
\end{abstract}

\section{INTRODUCTION}

Littlewood-Richardson coefficients arise in a variety of areas of mathematics and therefore not only knowing how to calculate them, but also knowing relations between them, is of importance. More precisely, given partitions $\lambda, \mu, \nu$, the Littlewood-Richardson coefficient $c_{\mu \nu}^{\lambda}$ arises most prominently in the following three places. First, in the representation theory of the symmetric group, given Specht modules $S^{\mu}$ and $S^{\nu}$ we have

$$
\left(S^{\mu} \otimes S^{\nu}\right) \uparrow^{S_{n}}=\bigoplus_{\lambda} c_{\mu \nu}^{\lambda} S^{\lambda} .
$$

Secondly, considering the cohomology $H^{*}(G r(k, n))$ of the Grassmannian, the cup product of Schubert classes $\sigma_{\mu}$ and $\sigma_{\nu}$ is given by

$$
\sigma_{\mu} \cup \sigma_{\nu}=\sum_{\lambda} c_{\mu \nu}^{\lambda} \sigma_{\lambda}
$$

Lastly, in the algebra of symmetric functions the skew Schur function $s_{\lambda / \mu}$ can be expressed in terms of the basis of Schur functions, $s_{\nu}$, via

$$
s_{\lambda / \mu}=\sum_{\nu} c_{\mu \nu}^{\lambda} s_{\nu}
$$

Consequently, knowledge about $c_{\mu \nu}^{\lambda}$ impacts a number of fields. Examples of knowledge gleaned so far about $c_{\mu \nu}^{\lambda}$ include a variety of ways to compute them, such as the Littlewood-Richardson rule [9, 16, 19, 20], inequalities among them that arise from studying eigenvalues of Hermitian matrices 8, instances when they evaluate to zero [12, and polynomiality properties that they satisfy [4, 7, 13. However, one natural aspect that has yet to be fully exploited is that of equivalence classes of

Received by the editors June 30, 2007 and, in revised form, November 15, 2007.

2000 Mathematics Subject Classification. Primary 05E05; Secondary 05E10, 20C30.

Key words and phrases. Jacobi-Trudi determinant, Hamel-Goulden determinant, ribbon, symmetric function, skew Schur function, Weyl module.

The second author was supported in part by the National Sciences and Engineering Research Council of Canada. 
equal coefficients. One way to approach this would be to use (1.2) and ask when two skew Schur functions are equal. This avenue is worth pursuing since it was recently shown that computing the coefficients $c_{\mu \nu}^{\lambda}$ is \#P-complete [11].

Returning to representation theory, there exist two polynomial representations of $G L_{N}(\mathbb{C})$ known as Schur modules and Weyl modules. These modules do not form a set of irreducible modules, and so a natural line of enquiry would be to ascertain when two of them are isomorphic. Since these modules are determined up to isomorphism by their characters, we simply need to discover when two characters are equal. It so happens that when the modules are indexed by skew diagrams $D$, then the characters are precisely the skew Schur function $s_{D}$ on $N$ variables. In this case we therefore need only determine when two skew Schur functions are equal.

The question of skew Schur function equality arises naturally in one other place: the algebra of symmetric functions. As implied earlier, the skew Schur functions are not a basis for the symmetric functions, and a question currently considered to be intractable is to find all relations among them. In [14] it was shown that the more specific goal of deriving all binomial syzygies between skew Schur functions could be attained by answering the question of equality. For this reason and those cited above, we will attempt to classify all skew Schur function equalities. In order to do this, we define the following equivalence relation.

Definition. For two skew diagrams $D$ and $D^{\prime}$ we say they are skew-equivalent if $s_{D}=s_{D^{\prime}}$ and denote this equivalence by $D \sim D^{\prime}$.

Our question of when two skew Schur functions are equal then reduces to classifying the equivalence classes of $\sim$.

It should be noted that we are not the first to investigate this equivalence. In 1] skew-equivalence was completely characterized for the subset of skew diagrams known as ribbons (or border strips or rim hooks). Their classification involved a certain composition of ribbons $\alpha$ and $\beta$ that forms a new ribbon $\alpha \circ \beta$. The idea behind composition operations is that they allow us to construct new equivalences from equivalences involving smaller skew diagrams. For example, the results in [1] tell us that if $\alpha \sim \alpha^{\prime}$ and $\beta \sim \beta^{\prime}$ are equivalences of ribbons, then $\alpha \circ \beta \sim$ $\alpha^{\prime} \circ \beta^{\prime}$. They were also able to show that the size of every equivalence class of $\circ$ is a specific power of 2 . The composition $\circ$ was generalised in [14] to include more general skew diagrams $D$ and yielded compositions $\alpha \circ D$ and $D \circ \beta$. A new composition of skew diagrams denoted by $\alpha \circ_{\omega} D$ for ribbons $\alpha, \omega$ and skew diagram $D$ was also introduced, as was the concept of ribbon staircases. These constructions successfully explained almost all skew-equivalences for skew diagrams with up to 18 cells, but unfortunately 6 skew-equivalences evaded the authors. In this paper we unify all the above constructions into one construction $D \circ_{W} E$ for skew diagrams $D, E$ and $W$. This composition not only provides us with an explanation for all skew-equivalences discovered to date, but it also suggests necessary and sufficient conditions for skew-equivalence. Furthermore, it affords us the possibility to conjecture that all equivalence classes are a specific power of 2 in size. More precisely, this paper is structured as follows.

In the next section we review the necessary preliminaries such as skew diagrams and symmetric functions, and recall two identities that will be crucial in our main proof. The first of these identities is the Hamel-Goulden determinant that was used with much success in [14] to determine skew-equivalence, and the second identity is the classical matrix theory result known as Sylvester's Determinantal Identity. In 
Section 3 we describe how to compose two skew diagrams $D$ and $E$ with respect to a third, $W$, to obtain $D \circ_{W} E$. For ribbons $\alpha, \beta, \omega$ and a skew diagram $D$ we discuss how our composition generalises the composition $\alpha \circ \beta$ of [1] and generalises the compositions $\alpha \circ D, D \circ \beta$ and $\alpha \circ_{\omega} D$ plus the notion of ribbon staircases found in 14. It is also in this section that we state our central theorem, Theorem 3.28. This theorem is the key to proving our sufficient condition for skew-equivalence, and the whole of Section 4 is devoted to its proof. Finally in Section 5 , as a consequence of Theorem 3.28, Theorem 5.3 gives our sufficient condition for skew-equivalence. We propose in Conjecture 5.7 that a closely related condition is necessary and sufficient for skew-equivalence, and that the size of every equivalence class is a specific power of 2. We also derive some conditions under which $D$ is skew-equivalent to its transpose in Proposition 5.1 and conjecture the that converse is also true.

\section{Preliminaries}

2.1. Diagrams. Before we embark on studying skew Schur functions, we need to recall the following combinatorial constructions. We say a partition, $\lambda$, of $n$ is a list of positive integers $\lambda_{1} \geq \lambda_{2} \geq \cdots \geq \lambda_{k}>0$ whose sum is $n$. We denote this by $\lambda \vdash n$, call $k=: \ell(\lambda)$ the length of $\lambda$, and call $n$ the size of $\lambda$, denoted by $|\lambda|$. For convenience we denote the unique partition of 0 by $\emptyset$. To every partition $\lambda$ we can associate a subset of $\mathbb{Z}^{2}$ called a diagram that consists of $\lambda_{i}$ left-justified cells in row $i$. By abuse of notation we also denote this diagram by $\lambda$. In the example below, the symbol $\times$ denotes a cell, although in what follows we may choose to denote cells by numbers, letters or boxes for further clarity.

Example 2.1.

$$
(3,2,2,1)=\begin{array}{ccc}
\times & \times & \times \\
\times & \times & \\
\times & \times \\
& \times &
\end{array} .
$$

Using this convention for constructing diagrams, we locate cells in the diagram by their row and column indices $(i, j)$, where $i \leq \ell(\lambda)$ and $j \leq \lambda_{1}$. Moreover, if a cell is contained in row $i$ and column $j$ of a diagram, then we say $c(i, j)=j-i$ is the content or diagonal of the cell. We will often use navigational terminology to refer to cells of a diagram. For example, the south (respectively east) border consists of those cells $(i, j)$ such that $(i+1, j)$ (resp. $(i, j+1))$ is not an element of the diagram, while the southeast border consists of those cells $(i, j)$ such that $(i+1, j+1)$ is not an element of the diagram. A cell $(i, j)$ is said to be strictly north of a cell $\left(i^{\prime}, j^{\prime}\right)$ if $i<i^{\prime}$, while $(i, j)$ is said to be one position northwest of $\left(i^{\prime}, j^{\prime}\right)$ if $(i, j)=\left(i^{\prime}-1, j^{\prime}-1\right)$.

Now consider two diagrams $\lambda$ and $\mu$ such that $\ell(\lambda) \geq \ell(\mu)$ and $\lambda_{i} \geq \mu_{i}$ for all $i \leq \ell(\mu)$, which we denote by $\mu \subseteq \lambda$. If we locate the cells of $\mu$ in the northwest corner of the set of cells of $\lambda$, then the skew diagram $\lambda / \mu$ is the array of cells contained in $\lambda$ but not in $\mu$, where $\lambda / \emptyset=\lambda$. As an example of a skew diagram we have

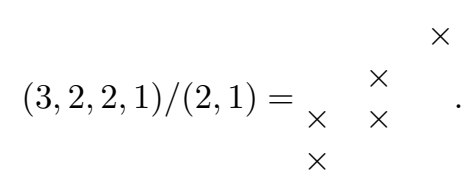


For convenience we will often refer to generic skew diagrams by capital letters such as $D$. As with partitions we will call the number of cells in $D$ the size of $D$ and denote it by $|D|$. We also consider two skew diagrams to be equal as subsets of the plane if one can be obtained from the other by the addition or deletion of empty rows or columns, or by vertical or horizontal translation.

Any subset of the cells of $D$ that itself forms a skew diagram is said to be a subdiagram of $D$. If two cells $(i, j)$ and $\left(i^{\prime}, j^{\prime}\right)$ satisfy $\left|i-i^{\prime}\right|+\left|j-j^{\prime}\right|=1$, then we say that they are adjacent, and we similarly say that two subdiagrams $D_{1}$ and $D_{2}$ are adjacent if $D_{1} \cap D_{2}=\emptyset$ and there exists a cell in $D_{1}$ adjacent to a cell in $D_{2}$. This concept will play a fundamental role in the pages to follow, but now we will use it to define what it means to be a connected skew diagram. A skew diagram is said to be connected if for every cell $d$ with another cell strictly north or east of it, there exists a cell adjacent to $d$ either to the north or to the east. A connected skew diagram is called a ribbon (or border strip or rim hook) if it does not contain the subdiagram $\lambda=(2,2)$.

Given any connected skew diagram $D$ there exist two natural subdiagrams of $D$, both of which are ribbons. The first is denoted by $n w_{D}$ and is the ribbon that starts at the southwesternmost cell of $D$, traverses the northwest border of $D$, and ends at the northeasternmost cell of $D$. The second is denoted by $s e_{D}$ and is the ribbon that starts at the southwesternmost cell of $D$, traverses the southeast border of $D$, and ends at the northeasternmost cell of $D$. A skew diagram closely related to $s e_{D}$ is $\widetilde{D}$, defined in set notation by $\widetilde{D}:=D \backslash s e_{D}$.

To close this subsection, we recall two symmetries on a skew diagram $D$. The first of these symmetries is the transpose or conjugate of $D$, denoted $D^{t}$, which is obtained by reflecting $D$ along the diagonal that runs from northwest to southeast through all cells with content 0 . The second is the antipodal rotation of $D$, denoted $D^{*}$, which is obtained by rotating $D$ by 180 degrees in the plane.

2.2. The algebra of symmetric functions. The algebra of symmetric functions has many facets to it, and in this section we review the pertinent details required for our results. More information on this fascinating algebra can be found in [10, 15, 17.

Let $\Lambda^{n}$ be the set of all formal power series $\mathbb{Z}\left[x_{1}, x_{2}, \ldots\right]$ in countably many variables that are homogeneous of degree $n$ in the $x_{i}$, and invariant under all permutations of the variables. Then the algebra of symmetric functions is

$$
\Lambda:=\bigoplus_{n \geq 0} \Lambda^{n}
$$

where $\Lambda_{0}=\operatorname{span}\{1\}=\mathbb{Z}$. It transpires that $\Lambda$ is a polynomial algebra in the complete symmetric functions, which are defined for all integers $r>0$ by

$$
h_{r}:=\Sigma_{1 \leq i_{1} \leq i_{2} \leq \cdots \leq i_{r}} x_{i_{1}} x_{i_{2}} \cdots x_{i_{r}},
$$

and $h_{0}=1$. To obtain a $\mathbb{Z}$-basis for $\Lambda$, let $\lambda=\left(\lambda_{1}, \ldots, \lambda_{k}\right)$ be a partition of $n$ and let

$$
h_{\lambda}:=h_{\lambda_{1}} \cdots h_{\lambda_{k}},
$$

for which we find $\left\{h_{\lambda}\right\}_{\lambda \vdash n}$ is a $\mathbb{Z}$-basis for $\Lambda^{n}$. However, for the reasons cited in the introduction, it is arguable that the most important $\mathbb{Z}$-basis of $\Lambda$ is that consisting of the Schur functions, which we now define as a subset of the skew Schur functions. 
Given a skew diagram $D$, we say that $T$ is a semistandard Young tableau if $T$ is a filling of the cells of $D$ with positive integers such that:

$\circ$ the entries in the rows weakly increase when read from west to east, and

o the entries in the columns strictly increase when read from north to south.

The skew Schur function $s_{D}$ is then

$$
s_{D}:=\sum_{T} x^{T}
$$

where the sum ranges over all semistandard Young tableaux of shape $D$, and

$$
x^{T}:=\prod_{(i, j) \in D} x_{T_{i j}} .
$$

Moreover, the skew Schur function is a ribbon Schur function if $D$ is a ribbon, and it is a $S$ chur function if for $D=\lambda / \mu$ we have that $\mu=\emptyset$. In this latter case we usually write $s_{D}=s_{\lambda}$, which yields another description of $\Lambda$ as $\Lambda=\bigoplus_{n>0} \Lambda^{n}$, where $\Lambda^{n}=\operatorname{span}\left\{s_{\lambda} \mid \lambda \vdash n\right\}$. Using (2.1) above, observe that the relationship between the basis of complete symmetric functions and the Schur functions is $h_{r}=s_{(r)}$, where $(r)$ denotes the diagram consisting of one row of $r$ cells for $r>0$. It follows from (1.1) and (1.2) that the skew Schur functions are Schur-positive, i.e. they can be written as a nonnegative linear combination of Schur functions. Moreover, products of Schur functions are also Schur-positive. This follows via the adjointness property with respect to the Hall inner product [10, Chapter 1, Equation (5.1)], which says that given partitions $\lambda, \mu, \nu$ with $\mu \subseteq \lambda$

$$
\left\langle s_{\lambda}, s_{\mu} s_{\nu}\right\rangle=\left\langle s_{\lambda / \mu}, s_{\nu}\right\rangle .
$$

Hence products of Schur functions and thus products of skew Schur functions are Schur-positive. This property of Schur-positivity will be useful to us later. However, it is a skew Schur function expansion into ribbon Schur functions that we wish to pursue in detail now.

2.3. Hamel-Goulden determinants. There are a number of ways of expressing a skew Schur function in terms of a matrix determinant involving ribbon Schur functions. For example, the Jacobi-Trudi determinant involves ribbons that are rows or columns, the Giambelli determinant involves hooks, and the Lascoux-Pragacz determinant involves certain more complex ribbons. However, the determinant we will describe generalises all of these, and since it was introduced in [5, it is known as the Hamel-Goulden determinant. Our description follows that of 3 .

If $D$ is a skew diagram, then a decomposition of $D$ is simply a partition of the elements of $D$ into disjoint subdiagrams of $D$. A ribbon decomposition is an ordered decomposition $\Pi=\left(\theta_{1}, \ldots, \theta_{m}\right)$ of $D$ into ribbons, and furthermore it is an outside (ribbon) decomposition if each $\theta_{i}$ is a ribbon whose southwesternmost (resp. northeasternmost) cell lies on either the west or south (resp. east or north) border of $D$. In this case we call $\theta_{i}$ an outside ribbon. Given an outside decomposition $\Pi$ of $D$ we say a cell $x \in \theta_{i}$ :

- goes north if the cell adjacent to $x$ to the north is also in $\theta_{i}$, or $x$ is the northeasternmost cell of $\theta_{i}$ and lies on the north border of $D$; and

- goes east if the cell adjacent to $x$ to the east is also in $\theta_{i}$, or $x$ is the northeasternmost cell of $\theta_{i}$ and lies on the east border of $D$. 
Observe that every cell in the same diagonal of $D$ either goes north or goes east with respect to $\Pi$. With this in mind the cutting strip $\theta(\Pi)$ of $\Pi$ is the unique ribbon occupying the same diagonals as $D$ such that a cell $x \in \theta(\Pi)$ goes north or goes east if and only if the cells in $D$ with content $c(x)$ go north or go east with respect to $\Pi$. Note that each ribbon $\theta_{i}$ naturally corresponds to the subdiagram of $\theta(\Pi)$ that contains the cells whose contents lie in the interval $\left[p\left(\theta_{i}\right), q\left(\theta_{i}\right)\right]$, where $p\left(\theta_{i}\right)$ is the content of the southwesternmost cell of $\theta_{i}$, and $q\left(\theta_{i}\right)$ is the content of the northeasternmost cell of $\theta_{i}$. Extending this notion, we define $\theta[p, q]$ to be the subdiagram of $\theta(\Pi)$ that contains the cells whose contents lie in the interval $[p, q]$ where:

$\circ \theta[q+1, q]=\emptyset$, the empty ribbon, and

○ $\theta[p, q]=$ undefined when $p>q+1$.

If we define

$$
\theta_{i} \# \theta_{j}:=\theta\left[p\left(\theta_{j}\right), q\left(\theta_{i}\right)\right],
$$

then the Hamel-Goulden determinant states that for any outside decomposition $\Pi=\left(\theta_{1}, \ldots, \theta_{m}\right)$ of a skew diagram $D$ we have

$$
s_{D}=\operatorname{det}\left(s_{\theta_{i} \# \theta_{j}}\right)_{i, j=1}^{m},
$$

where $s_{\emptyset}=1$ and $s_{\text {undefined }}=0$. We call $\left(s_{\theta_{i} \# \theta_{j}}\right)_{i, j=1}^{m}$ the Hamel-Goulden matrix.

Example 2.2. If $D=(3,3,3,1) /(1)$, then one possible outside decomposition $\Pi=\left(\theta_{1}, \theta_{2}\right)$ is shown below. The cells in $\theta_{i}$ are labelled by $i$, and the cutting strip $\theta(\Pi)$ and identification of the ribbons $\theta_{i}$ with intervals of contents within $\theta(\Pi)$ are also shown.

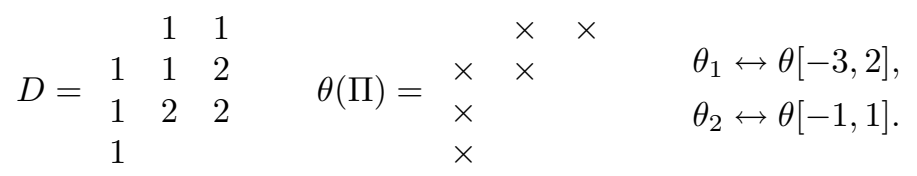

The Hamel-Goulden determinant is then

$$
\begin{aligned}
& s_{D}=\operatorname{det}\left[\begin{array}{ll}
s_{\theta[-3,2]} & s_{\theta[-1,2]} \\
s_{\theta[-3,1]} & s_{\theta[-1,1]}
\end{array}\right]
\end{aligned}
$$

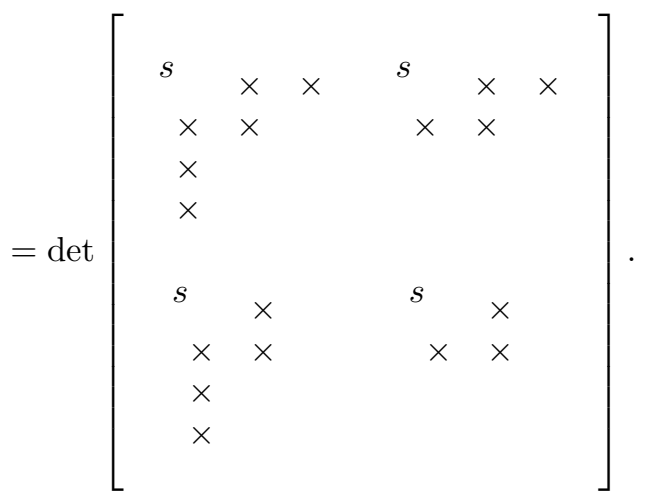

Observe that in this case $\theta(\Pi)=\theta_{1}=n w_{D}$. However, this is not true in general but is a property of the outside decomposition we chose, which we define next. 
Definition 2.3. Given a connected skew diagram $D$ the southeast decomposition is an outside decomposition of $D$ that is unique up to reordering. We construct it by choosing the first ribbon to be $s e_{D}$. Now consider $D$ with $s e_{D}$ removed and iterate the procedure on the remaining skew diagram. If this skew diagram is no longer connected, then iterate on each of the connected components.

We can similarly define the northwest decomposition by utilising $n w_{D}$.

Observe that the above example is a northwest decomposition, and it is straightforward to see that in general with a northwest decomposition, $\Pi$, of a skew diagram $D$, the cutting strip is $\theta(\Pi)=n w_{D}$. Similarly, if we had used a southeast decomposition, then $\theta(\Pi)=s e_{D}$. A third outside decomposition that will be useful later is the horizontal or Jacobi-Trudi decomposition in which $\theta_{i}$ is simply row $i$ of the skew diagram $D$.

The last of our preliminaries is the following result, known as Sylvester's Determinantal Identity, and can be found in standard matrix theory references, such as 6]. This identity will serve a pivotal role in the proof of Theorem 3.28, where the matrix in question will be a Hamel-Goulden matrix. If $M$ is an $n$-by- $n$ matrix, and $A, B \subseteq\{1, \ldots, n\}$, then we let $M[A, B]$ denote the submatrix of $M$ consisting of those entries $(i, j)$ of $M$ having $i \in A$ and $j \in B$.

Theorem 2.4. Let $M$ be an n-by-n matrix, and let $S \subseteq\{1, \ldots, n\}$. Define a matrix $\operatorname{Syl}(M, S)$, with rows and columns indexed by $\{1, \ldots, n\} \backslash S$, by

$$
\operatorname{Syl}(M, S)_{i, j}=\operatorname{det} M[S \cup\{i\}, S \cup\{j\}]
$$

for $i, j \notin S$. Then

$$
(\operatorname{det} M)(\operatorname{det} M[S, S])^{n-|S|-1}=\operatorname{det} \operatorname{Syl}(M, S) .
$$

In the case when $S=\{2, \ldots, n-1\},(2.3)$ is known as the Desnanot-Jacobi Identity and is equivalent to Dodgson's condensation formula.

\section{COMPOSITIONS OF SKEW DIAGRAMS}

It is now time to recall our equivalence relation that was defined for ribbons in [1] and generalised in [14.

Definition 3.1. For two skew diagrams $D$ and $D^{\prime}$ we say they are skew-equivalent if $s_{D}=s_{D^{\prime}}$, and denote this equivalence by $D \sim D^{\prime}$.

The goal of this paper is to classify skew-equivalence by a condition that is both necessary and sufficient. Fortunately the number of skew-equivalences we need to classify is greatly reduced due to

Proposition 3.2 ([14, Section 6]). Understanding the equivalence relation $\sim$ on all skew diagrams is equivalent to understanding $\sim$ among connected skew diagrams.

Consequently, we will henceforth assume that all skew diagrams are connected unless otherwise stated.

We will also make use of the following necessary conditions for skew-equivalence.

Theorem 3.3. For skew diagrams $D$ and $D^{\prime}$, if $D \sim D^{\prime}$, then we have:

(i) The number of cells in $D$ equals the number of cells in $D^{\prime}$.

(ii) The number of rows in $D$ equals the number of rows in $D^{\prime}$.

(iii) $|\widetilde{D}|=\left|\widetilde{D^{\prime}}\right|$. 
Proof. $\quad$ (i) This comes from the definition of $s_{D}$ in terms of tableaux (2.1).

(ii) This follows immediately from [14, Proposition 6.2(ii)], where it was shown that the multisets of row lengths of $D$ and $D^{\prime}$ are equal.

(iii) There exists an involution $\omega$ on $\Lambda$ such that $\omega\left(s_{D}\right)=s_{D^{t}}$ [10, Chapter 1, Equation (5.6)] and hence $D^{t} \sim\left(D^{\prime}\right)^{t}$. By the previous part this implies that the number of columns in $D$ equals the number of columns in $D^{\prime}$. Since $\left|s e_{D}\right|+1$ is the number of rows of $D$ plus the number of columns of $D$, the result now follows by the first part.

Our approach throughout will be to use known skew-equivalences to construct skew-equivalences for larger skew diagrams. Our basic building blocks will be the skew-equivalences of the following proposition, which is not hard to prove using the symmetry of $s_{D}$ and its definition in terms of tableaux (2.1).

Proposition 3.4 ([17, Exercise 7.56(a)]). For any skew diagram $D, D^{*} \sim D$.

The other main ingredient, and the focus of this paper, is a way to put these building blocks together to construct more complex skew-equivalences. More specifically, we wish to define a notion of composition $D \circ E$ for skew diagrams $D$ and $E$. Then if $D \sim D^{\prime}$ and $E \sim E^{\prime}$, our hope will be that $D \circ E \sim D^{\prime} \circ E^{\prime}$. Since we wish to generalise and unify the three main operations of [14, some care needs to be taken when defining our composition operation, and some preliminary work is in order.

Definition 3.5. Given skew diagrams $W$ and $E$, we say that $W$ lies in the top (resp. bottom) of $E$ if $W$ appears as a connected subdiagram of $E$ that includes the northeasternmost (resp. southwesternmost) cell of $E$.

Given two skew diagrams $E_{1}$ and $E_{2}$ and a skew diagram $W$ lying in the top of $E_{1}$ and the bottom of $E_{2}$, the amalgamation of $E_{1}$ and $E_{2}$ along $W$, denoted by $E_{1} \amalg_{W} E_{2}$, is the new skew diagram obtained from the disjoint union of $E_{1}$ and $E_{2}$ by identifying the copy of $W$ in the top of $E_{1}$ with the copy of $W$ in the bottom of $E_{2}$.

If $W$ lies in both the top and bottom of $E$, then we will let $W_{n e}$ (resp. $W_{s w}$ ) denote the copy of $W$ in the top (resp. bottom) of $E$. We can also define

$$
E^{\amalg_{W} m}=\underbrace{E \amalg_{W} E \amalg_{W} \cdots \amalg_{W} E}_{m \text { factors }}:=\left(\cdots\left(\left(E \amalg_{W} E\right) \amalg_{W} E\right) \amalg_{W} \cdots\right) \amalg_{W} E .
$$

Example 3.6. The skew diagram $E$ given by

$$
E=\begin{array}{ccccccccc} 
& & & & \times & \times & \times & \times & \times \\
& \times & \times & \times & \times & & & \\
& \times & \times & \times & \times & & & &
\end{array}
$$

has

$$
W=\underset{\times}{\times} \times
$$


lying in its top and bottom. We see that

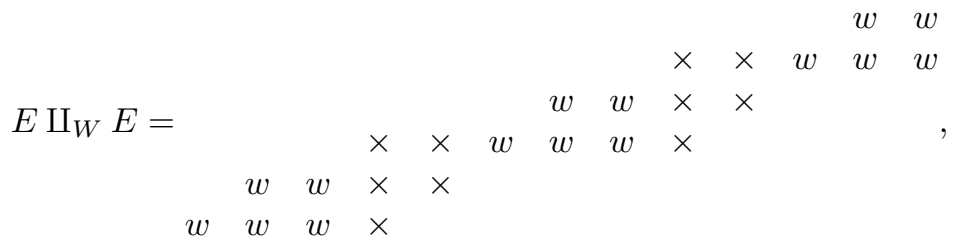

where we use the symbol $w$ to denote the cells of copies of $W$. Notice that $V=$ $\underset{\times}{\times}{ }^{x}$ also lies in the top and bottom of $E$, and that $E \amalg_{V} E$ is the same skew diagram as $E \amalg_{W} E$.

Example 3.7. For complete generality, we will also say that when $W=\emptyset, W$ lies in the top and bottom of any skew diagram $E$. In this case, we will identify $W_{s w}$ with the west edge of the southwesternmost cell of $E$. Similarly, we will identify $W_{n e}$ with the east edge of the northeasternmost cell of $E$. For example, if $E=(3,3,2) /(1)$, then $W_{s w}$ and $W_{n e}$ would be identified with the thicker edges as shown:

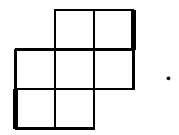

Then $E \amalg_{\emptyset} E$ is the skew diagram $(6,6,5,3,2) /(4,3,1)$.

Now is a good time to introduce some assumptions on $E$ and $W$ that we will need for our results to hold.

Hypotheses 3.8. Suppose that $E$ is a skew diagram having $W$ lying in its top and bottom. We assume that $E$ and $W$ satisfy the following conditions:

(I) $W$ is maximal in the following sense: there does not exist a skew diagram $W^{\prime} \supsetneq W$ that occupies the same set of diagonals as $W$ and that also lies in the top and bottom of $E$.

(II) $W_{n e}$ and $W_{s w}$ are separated by at least one diagonal. In other words, there is at least one diagonal between $W_{n e}$ and $W_{s w}$ that intersects neither $W_{n e}$ nor $W_{s w}$.

(III) The complement in $E$ of either copy of $W$ is a connected skew diagram.

Remark 3.9. Analogues of Hypotheses [I and \II are also necessary for the results

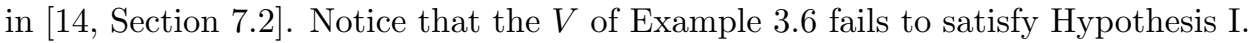
(It also fails to satisfy Hypothesis III) As we saw, however, $E \amalg_{V} E=E \amalg_{W} E$, and it will be apparent from our definition of the composition operation that $D \circ E$ for any $D$ is the same whether we work with $V$ or $W$. Therefore, we lose no generality when we impose Hypothesis $\square$-it will just make the statements of some of our results simpler.

Hypotheses $\Pi$ and $\llbracket$ tell us much about the structure of $E$. Let $O$ denote the subdiagram of $E$ that results when we delete both copies of $W$. We will write $E=W O W$ to mean that $W$ lies in the top and bottom of $E$ and that $O$ is the 
subdiagram of $E$ that results when we delete both copies of $W$. Since $E$ is assumed to be connected, Hypotheses $\amalg$ and $\amalg$ tell us that $O$ is a non-empty connected skew diagram.

Let us say that the lower (resp. upper) copy of $W$ is horizontally attached to $O$ if the southwesternmost (resp. northeasternmost) cell of $O$ has a cell of $W$ one position to its west (resp. east). Similarly, we say that the lower (resp. upper) copy of $W$ is vertically attached to $O$ if the southwesternmost (resp. northeasternmost) cell of $O$ has a cell of $W$ one position to its south (resp. north). Since $W$ is a skew diagram and $E$ is connected, each copy of $W$ in $E$ is either horizontally or vertically attached to $O$, but not both. Therefore, we are in one of the following four cases:

(a) Both copies of $W$ are horizontally attached to $O$, written $E=W \rightarrow O \rightarrow W$.

(b) Both copies of $W$ are vertically attached to $O$, written $E=W \uparrow O \uparrow W$.

(c) The lower copy of $W$ is horizontally attached to $O$, while the upper copy of $W$ is vertically attached to $O$, written $E=W \rightarrow O \uparrow W$.

(d) The lower copy of $W$ is vertically attached to $O$, while the upper copy of $W$ is horizontally attached to $O$, written $E=W \uparrow O \rightarrow W$.

We are almost ready to define composition of general skew diagrams. One issue that lengthens the definition of the composition of $D$ and $E$ with respect to $W$ is that the definition varies according to the cases (a), (b), (c) and (d) above. As justification for this variation, consider the following diagrams that can be created, starting with two copies $E_{1}$ and $E_{2}$ of $E$ :

(A) Position $E_{2}$ so that the lower copy of $W$ in $E_{2}$ is one position northwest of the upper copy of $W$ in $E_{1}$.

(B) Position $E_{2}$ so that the lower copy of $W$ in $E_{2}$ is one position southeast of the upper copy of $W$ in $E_{1}$.

(C) Form $E_{1} \amalg_{W} E_{2}$ and translate an extra copy of $W$ one position southeast from $E_{1} \cap E_{2}$.

(D) Form $E_{1} \amalg_{W} E_{2}$ and translate an extra copy of $W$ one position northwest from $E_{1} \cap E_{2}$.

The key observation is that in each of the four cases (a), (b), (c) and (d), exactly one of these four diagrams is a skew diagram, namely the diagram with the corresponding letter label. This observation effectively consists of sixteen assertions, and we leave their checking as an exercise for the reader that will reinforce the ideas introduced so far. In each of the four cases (a), (b), (c) and (d), we let $E_{1} \cdot{ }_{W} E_{2}$ denote the skew diagram constructed in (A), (B), (C) and (D) respectively. See Figure 1 for an illustration.

Remark 3.10. We see that there is a fundamental difference between the set-up for the cases $W \rightarrow O \rightarrow W, W \uparrow O \uparrow W$ and the cases $W \rightarrow O \uparrow W, W \uparrow O \rightarrow W$. In a certain sense, this difference is to be expected, since it turns out that $W \rightarrow O \rightarrow W$ and $W \uparrow O \uparrow W$ are involved in generalising the composition and amalgamated composition operations of [14], while $W \rightarrow O \uparrow W$ and $W \uparrow O \rightarrow W$ are involved in generalising the ribbon staircase operation. The real strength of our framework will be highlighted by the statements of the results that follow, where all four cases can be treated as one.

We are finally ready to define the composition of general skew diagrams.

Definition 3.11. For skew diagrams $D, E$ with $E=W O W$ subject to Hypotheses 3.8, we define the composition $D \circ_{W} E$ with respect to $W$ as follows. Every 

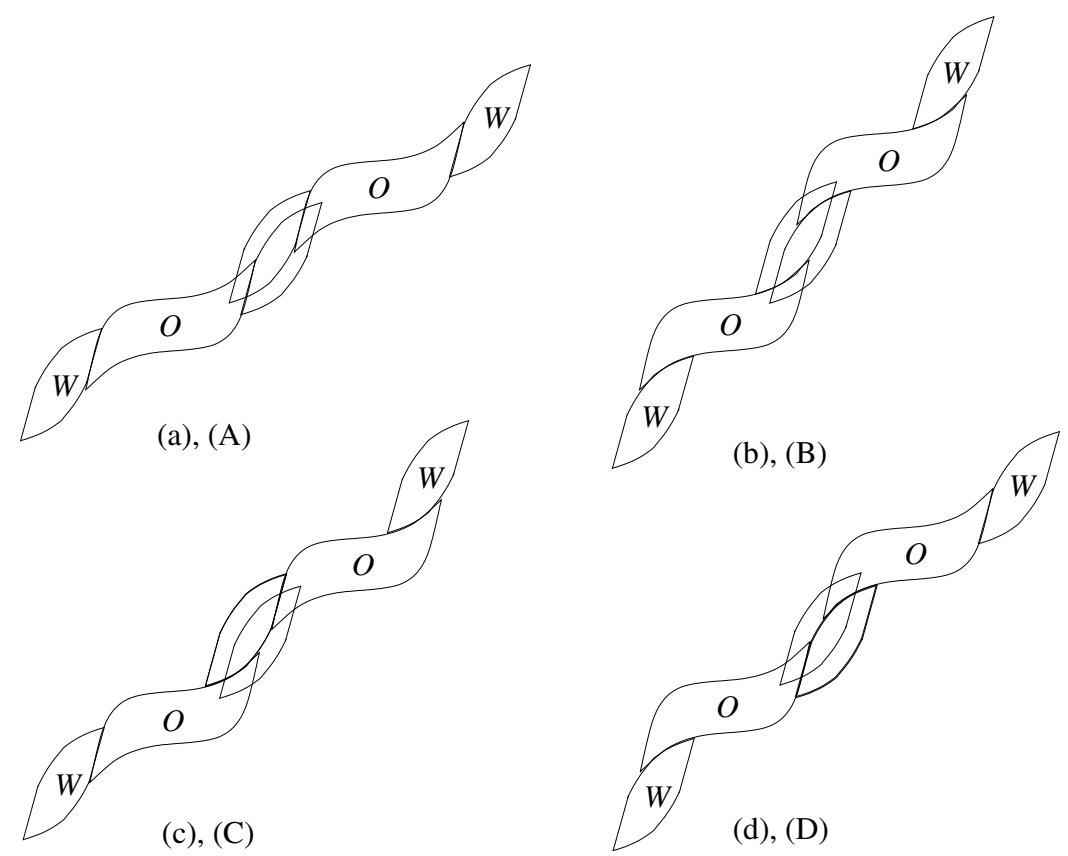

FiguRE $1 . E_{1} \cdot W E_{2}$ in the four cases

cell $d$ of $D$ will contribute a copy of $E$, denoted $E_{d}$, in the plane. The set of copies $\left\{E_{d} \mid d \in D\right\}$ are combined according to the following rules:

(a), (b) Suppose $E=W \rightarrow O \rightarrow W$ or $E=W \uparrow O \uparrow W$.

(i) If $d$ is one position west of $d^{\prime}$ in $D$, then $E_{d}$ and $E_{d^{\prime}}$ appear in the form $E_{d} \amalg_{W} E_{d^{\prime}}$.

(ii) If $d$ is one position south of $d^{\prime}$ in $D$, then $E_{d}$ and $E_{d^{\prime}}$ appear in the form $E_{d} \cdot W E_{d^{\prime}}$.

(c), (d) If $E=W \rightarrow O \uparrow W$, then we consider the northwest ribbon decomposition of $D$, while if $E=W \uparrow O \rightarrow W$, then we consider the southeast ribbon decomposition of $D$.

(i) If $d$ is one position west of $d^{\prime}$ on the same ribbon in $D$, then $E_{d}$ and $E_{d^{\prime}}$ appear in the form $E_{d} \amalg_{W} E_{d^{\prime}}$.

(ii) If $d$ is one position south of $d^{\prime}$ on the same ribbon in $D$, then $E_{d}$ and $E_{d^{\prime}}$ appear in the form $E_{d} \cdot W E_{d^{\prime}}$.

(iii) If $d$ is one position southeast of $d^{\prime}$ in $D$, then $E_{d}$ appears one position southeast of $E_{d^{\prime}}$.

Additionally, we will use the convention that $\emptyset \circ_{W} E=W$ and that $D \circ_{W} E$ is undefined when $D$ is undefined.

Example 3.12. Identifying the cells of $D$ with integers, and labelling the cells of the copies of $W$ in $E$ with the letter $w$, suppose

$$
D=\begin{array}{ll}
1 & 2 \\
3 & 4
\end{array} \text { and } E=\begin{array}{r}
w \\
w
\end{array} \times \quad \times \quad \begin{aligned}
& w \\
&
\end{aligned}
$$


Then $E=W \rightarrow O \rightarrow W$, and so $D \circ_{W} E$ is the skew diagram

$$
\begin{array}{ccccccc} 
& & & & & & 2 \\
1 & 1 & 1 & \times & \times & & \\
1 & \times & 4 & 4 & 4 & & \\
3 & \times & 4 & & & &
\end{array}
$$

where a cell is labelled by $\times$ if it is an element of $E_{d}$ for more than one $d \in D$, and otherwise is labelled by $d$ when it is an element of $E_{d}$.

Alternatively, if

$$
E=\begin{array}{lll} 
& & w \\
w & \times & \times \\
w & \times
\end{array},
$$

then $E=W \rightarrow O \uparrow W$, and so $D \circ_{W} E$ is the skew diagram

$\begin{array}{lllllll} & & & & & & 2 \\ & & & & & & 2 \\ & & & & & 2 & 2 \\ & & & & \times & \times & \\ & & & & & & \\ & & & 1 & 1 & 4 & \\ & & \times & \otimes & 4 & 4 & \\ 3 & 3 & 3 & \otimes & 4 & & \\ 3 & 3 & & & & & \end{array}$

where $\otimes$ denotes an element of both $E_{4}$ and $E_{3} \cdot W E_{1}$.

Example 3.13. If $W$ is empty, then referring to Example 3.7, it is natural to consider $E$ to be of the form $W \rightarrow O \rightarrow W$. If at least one of $D$ and $E$ is a ribbon, $\circ_{\emptyset}$ becomes the composition denoted simply by $\circ$ in [14. When both $D$ and $E$ are ribbons, $D \circ_{\emptyset} E$ also corresponds to $D \circ E$ of $[1$. When neither $D$ nor $E$ is a ribbon, $\circ_{\emptyset}$ behaves like $\circ$, except that we allow overlaps to occur among copies of $E$. To see this in action, take $D$ as in the previous example, and let $E=\underset{\times}{\times} \underset{\times}{\times}$. Then

$$
D \circ_{\emptyset} E=\begin{array}{rrrrr} 
& & & 2 & 2 \\
& 1 & 1 & 2 & 2 \\
& 1 & \times & 4 & \\
3 & 3 & 4 & 4 & \\
3 & 3 & & &
\end{array} .
$$

We note in passing that this is the same skew diagram that appears in [14, Remark 7.10], and was the first motivating example for the work of the current article.

Example 3.14. The previous example demonstrates how one of the three main operations of [14] is obtained as a special case of our composition operation. The other two operations are also obtained as special cases: the amalgamated composition operation corresponds to certain cases of $D \circ_{W} E$ with $D$ and $W$ non-empty ribbons and $E$ of the form $W \rightarrow O \rightarrow W$ or $W \uparrow O \uparrow W$. On the other hand, if $E$ is a ribbon of the form $W \rightarrow O \uparrow W$ or $W \uparrow O \rightarrow W$, then $D \circ_{W} E$ is a ribbon staircase construction. 
Remark 3.15. We see in Definition 3.11 that $E=W \rightarrow O \uparrow W$ is associated with the northwest ribbon decomposition of $D$, while $E=W \uparrow O \rightarrow W$ is associated with the southeast ribbon decomposition. We remark that $E=W \rightarrow O \rightarrow W$ and $E=$ $W \uparrow O \uparrow W$ should both be associated with the horizontal ribbon decomposition. Indeed, (i) above for the (a), (b) case could equivalently state that if $d$ is one position west of $d^{\prime}$ on the same ribbon of the horizontal decomposition of $D$, then $E_{d}$ and $E_{d^{\prime}}$ appear in the form $E_{d} \amalg_{W} E_{d^{\prime}}$. This is the same rule as (i) for the (c), (d) case. More importantly, our proof of Theorem 3.28 for $E=W \rightarrow O \rightarrow W$ and $E=W \uparrow O \uparrow W$ will exploit the horizontal decomposition of $D$, in the same way that our proof for $E=W \rightarrow O \uparrow W$ and $E=W \uparrow O \rightarrow W$ will exploit the northwest and southeast decompositions.

When $E=W \rightarrow O \uparrow W$, we have defined $D \circ_{W} E$ in terms of the northwest decomposition of $D$. Before proceeding, we give an alternative definition of $D \circ_{W} E$, this time in terms of the southeast decomposition of $D$. This is a necessary tool for proving Lemma 3.19(iii) below. The reader that is focussing solely on the statements of the main results can safely skip the next definition, example and lemma.

First, we will give an alternative definition of $D \circ_{W} E$ that highlights the way in which its structure is affected by the extra copies of $W$ that arise from copies of $E$ that are in the form $E_{d} \cdot W E_{d^{\prime}}$. Let $W_{1}$ denote the extra copy of $W$ that is added to $E_{d} \amalg_{W} E_{d^{\prime}}$ to form $E_{d} \cdot W E_{d^{\prime}}$. We observe that $W_{1}$ will be covered by a copy $E_{d^{\prime \prime}}$ for some $d^{\prime \prime} \in D$ in many situations. Specifically, if $d^{\prime}$ has a cell $d^{\prime \prime}$ one position to its southeast, then $W_{1}$ will be covered by $E_{d^{\prime \prime}}$. Therefore, we could also define $D \circ_{W} E$ as follows.

Definition 3.16. For skew diagrams $D$ and $E$ with $E$ of the form $W \rightarrow O \uparrow W$, we define $D \circ_{W} E$ as follows. Every cell $d$ of $D$ will contribute a copy of $E$, denoted $E_{d}$, in the plane. Considering the northwest ribbon decomposition of $D$, we position the set of copies $\left\{E_{d} \mid d \in D\right\}$ according to the following rules:

(i) If $d$ is one position west or south of $d^{\prime}$ on the same ribbon in $D$, then $E_{d}$ and $E_{d^{\prime}}$ appear in the form $E_{d} \amalg_{W} E_{d^{\prime}}$.

(ii) If $d$ is one position southeast of $d^{\prime}$ in $D$, then $E_{d}$ appears one position southeast of $E_{d^{\prime}}$.

Furthermore, if $d$ is one position south of $d^{\prime}$ on the same ribbon in $D$ and both $d$ and $d^{\prime}$ are elements of $s e_{D}$, then add a copy of $W$ one position southeast of $E_{d} \cap E_{d^{\prime}}$.

Example 3.17. Suppose

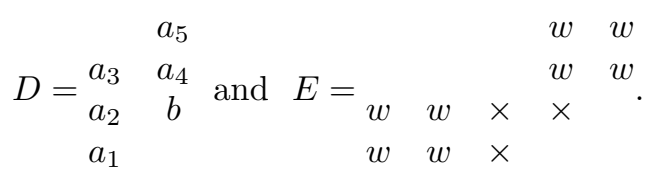

Then a representative diagram of $D \circ_{W} E$ is shown in Figure 2, The normal lines represent the contribution from the $a_{i}$ ribbon; the dotted lines represent $E_{b}$, while the bold lines represent the extra copies of $W$.

When $E=W \rightarrow O \uparrow W$, we are now in a position to give a definition of $D \circ_{W} E$ in terms of the southeast decomposition of $D$, as promised. It should be compared with the relevant part of Definition 3.11, 


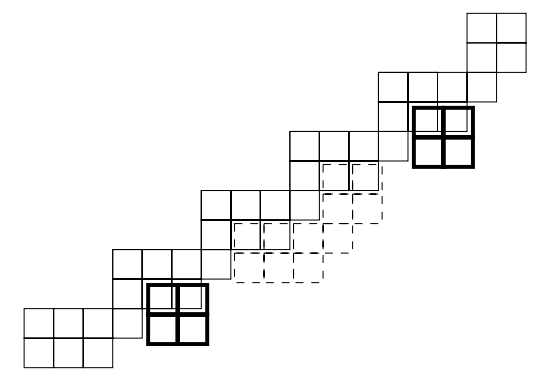

Figure 2. The real contribution from extra copies of $W$

Lemma 3.18. For skew diagrams $D$ and $E$ with $E$ of the form $W \rightarrow O \uparrow W$, suppose we define a diagram $D \star_{W} E$ as follows. Every cell $d$ of $D$ will contribute a copy of $E$, denoted $E_{d}$, in the plane. Considering the southeast decomposition of $D$, we position the set of copies $\left\{E_{d} \mid d \in D\right\}$ according to the following rules:

(i) If $d$ is one position west of $d^{\prime}$ on the same ribbon in $D$, then $E_{d}$ and $E_{d^{\prime}}$ appear in the form $E_{d} \amalg_{W} E_{d^{\prime}}$.

(ii) If $d$ is one position south of $d^{\prime}$ on the same ribbon in $D$, then $E_{d}$ and $E_{d^{\prime}}$ appear in the form $E_{d} \cdot W E_{d^{\prime}}$.

(iii) If $d$ is one position northwest of $d^{\prime}$ in $D$, then $E_{d}$ appears one position southeast of $E_{d^{\prime}}$.

Then $D \star_{W} E=D \circ_{W} E$.

Proof. In the same way that we extracted Definition 3.16 from Definition 3.11 we could extract an analogue of Definition 3.16 from the definition of $D \star_{W} E$. This analogue states that, considering the southeast ribbon decomposition of $D$, we construct $D \star_{W} E$ by positioning the set of copies $\left\{E_{d} \mid d \in D\right\}$ according to the following rules:

(i) If $d$ is one position west or south of $d^{\prime}$ on the same ribbon in $D$, then $E_{d}$ and $E_{d^{\prime}}$ appear in the form $E_{d} \amalg_{W} E_{d^{\prime}}$.

(ii) If $d$ is one position northwest of $d^{\prime}$ in $D$, then $E_{d}$ appears one position southeast of $E_{d^{\prime}}$.

Furthermore, if $d$ is one position south of $d^{\prime}$ on the same ribbon in $D$ and both $d$ and $d^{\prime}$ are elements of $n w_{D}$, then add a copy of $W$ one position southeast of $E_{d} \cap E_{d^{\prime}}$.

Thinking in terms of Definition 3.16 and this analogue, it is easy to see that an outside ribbon of size $l$ in $D$ contributes $E^{\amalg_{W} l}$ to $D \circ_{W} E$ and $D \star_{W} E$. Our proof now divides into two parts. We will first show that these contributions from the ribbons give the same result, whether we work with the northwest decomposition, as in $D \circ_{W} E$, or with the southeast decomposition, as in $D \star_{W} E$. To finish, we show that the effect of the extra copies of $W$ from the final sentence of Definition 3.16 on $D \circ_{W} E$ is the same as the effect of the extra copies of $W$ from the analogue on $D \star_{W} E$.

It is clear that $n w_{D}$ and $s e_{D}$ have the same size $l_{1}$, and so they will contribute the same $E_{1}=E^{\amalg_{W} l_{1}}$ to $D \circ_{W} E$ and $D \star_{W} E$ respectively. Furthermore, we can see that $D \backslash n w_{D}$ and $D \backslash s e_{D}$ are the same skew diagram $D_{1}$. It will not affect our argument that $D_{1}$ need not be connected. Repeating this process, we could remove 
a maximal size ribbon $r_{n w}$, that is an element of the northwest decomposition of $D$, from the northwest border of some connected component $D_{1}^{\prime}$ of $D_{1}$. We could also remove a maximal size ribbon $r_{s e}$, that is an element of the southeast decomposition of $D$, from $D_{1}^{\prime}$. Again, removing either $r_{n w}$ and $r_{s e}$ from $D_{1}^{\prime}$ results in the same skew diagram $D_{2}$. The key point is that $r_{n w}$ and $r_{s e}$ have the same size $l_{2}$ so, while they aren't in general the same ribbon, they contribute the same $E_{2}=E^{\amalg_{W} l_{2}}$ to $D \circ_{W} E$ and $D \star_{W} E$ respectively. Furthermore, since $r_{n w}$ and $r_{s e}$ have the same set of contents, the copies of $E_{2}$ will have the same positions relative to the copies of $E_{1}$.

Repeating this process, we will see that the contributions of the form $E^{\amalg_{W} l}$ to $D \circ_{W} E$ from the ribbons of the northwest decomposition will be the same, and have the same relative positions, as the analogous contributions to $D \star_{W} E$ from the ribbons of the southeast decomposition.

It remains to show that the extra copies of $W$ will be the same in both cases. This amounts to showing that the following are equivalent:

(1) There exists $d$ with content $i$ one position south of $d^{\prime}$ on the same ribbon of the northwest decomposition of $D$, and both $d$ and $d^{\prime}$ are elements of $s e_{D}$.

(2) There exists $c$ with content $i$ one position south of $c^{\prime}$ on the same ribbon of the southeast decomposition of $D$, and both $c$ and $c^{\prime}$ are elements of $n w_{D}$.

Suppose (1). Since $D$ is a skew diagram, and since $d$ and $d^{\prime}$ are on the same ribbon of the northwest decomposition of $D$, we see that $d \in n w_{D}$ if and only if $d^{\prime} \in n w_{D}$. If $d, d^{\prime} \in n w_{D}$, then we conclude (2), since $d$ and $d^{\prime}$ are on the same ribbon of the southeast decomposition of $D$. If $d, d^{\prime} \notin n w_{D}$, then there exist cells $e$ and $e^{\prime}$ one position northwest of $d$ and $d^{\prime}$ respectively. We see that since $d$ and $d^{\prime}$ are on the same ribbon in both the northwest and southeast decompositions of $D$, the same must apply to $e$ and $e^{\prime}$. For the same reasons as before, $e \in n w_{D}$ if and only if $e^{\prime} \in n w_{D}$. Repeating this process, working in a northwesterly direction, we will eventually arrive at $c$ and $c^{\prime}$ with the required properties. Since the process is clearly reversible, the result follows.

Readers may wish to check their understanding of the definition of $D \circ_{W} E$ by filling in the details in the proof of the following lemma. Part (i) is of obvious importance, while (ii) and (iii) will save us much effort in the proof of Theorem 3.28 ,

Lemma 3.19. For skew diagrams $D$ and $E=W O W$ we have:

(i) $D \circ_{W} E$ is a skew diagram.

(ii) $\left(D \circ_{W} E\right)^{*}=D^{*} \circ_{W^{*}} E^{*}$.

(iii) If $W \neq \emptyset$, then $\left(D \circ_{W} E\right)^{t}=D^{*} \circ_{W^{t}} E^{t}$.

Proof. (i) We can prove this by induction on the number $r$ of ribbons in the appropriate ribbon decomposition of $D$. Suppose $E=W \rightarrow O \rightarrow W$ or $E=W \uparrow O \uparrow W$. If $r=1$, then $D \circ_{W} E$ is just $E^{\amalg_{W}|D|}$. For $r>1$, the result follows from the fact that both $E \cdot W E$ and $D$ are skew diagrams. Now suppose $E=W \rightarrow O \uparrow W$ or $E=W \uparrow O \rightarrow W$. If $r=1$, then $D \circ_{W} E$ is $E^{\amalg_{W}|D|}$ with some copies of $W$ added as appropriate. The result will be a skew diagram since $E \cdot{ }_{W} E$ is a skew diagram. Now suppose $r>1$. First observe how the ribbons of the northwest/southeast decomposition of $D$ "nest" in each other. We can check that the contributions to $D \circ_{W} E$ of the various ribbons of the northwest/southeast decomposition of $D$ nest 
with each other in an analogous way. In the same way that $E \cdot{ }_{W} E$ is a skew diagram, we conclude that $D \circ_{W} E$ is a skew diagram.

(ii) We omit the proof since this identity is straightforward to check using Definition 3.11. Observe, though, that if $E=W \rightarrow O \uparrow W$, then $E^{*}=$ $W^{*} \uparrow O^{*} \rightarrow W^{*}$.

(iii) Notice that if $E=W \rightarrow O \rightarrow W$, then $E^{t}=W^{t} \uparrow O^{t} \uparrow W^{t}$. We omit the proof of the cases $E=W \rightarrow O \rightarrow W$ and $E=W \uparrow O \uparrow W$, since then the identity is checked easily, as in (ii). We next consider the case when $E=W \rightarrow O \uparrow W$. The ribbons of the northwest decomposition of $D$ correspond exactly to the ribbons of the southeast decomposition of $D^{*}$. Comparing Definition 3.11 in the case $E=W \rightarrow O \uparrow W$ with the definition of $D \circ_{W} E$ from the statement of Lemma 3.18 yields the result. If $E=W \uparrow O \rightarrow W$, then the proof is similar, since we could develop an appropriate analogue of Lemma 3.18 .

We can now work directly towards the statement of Theorem 3.28, which expresses $s_{D \circ_{W} E}$ in terms of $s_{D}$ and $s_{E}$, and thus serves as the foundation for all our skew-equivalence proofs. As mentioned in Example 3.13, a feature of our definition of $D \circ_{W} E$ is that we allow overlaps among the copies of $E$, whereas composition operations in prior work do not. At some point, we must obtain an understanding of, and account for, these overlaps. This motivates the following definition of the skew diagrams $\bar{W}$ and $\bar{O}$.

Definition 3.20. Consider the infinite skew diagram

$$
\boldsymbol{E}:=E^{\amalg_{W} \infty}=\cdots \amalg_{W} E \amalg_{W} E \amalg_{W} \cdots .
$$

For every copy $O_{1}$ of $O$ in $\boldsymbol{E}$ we define

$$
\overline{O_{1}}=\left\{(i, j) \in O_{1} \mid(i+1, j+1) \in O_{1}\right\} .
$$

For every copy $W_{1}$ of $W$ we define

$$
\overline{W_{1}}=\left\{(i, j) \in \boldsymbol{E} \mid(i+1, j+1) \in W_{1}\right\} \cup\left\{(i, j) \in W_{1} \mid(i+1, j+1) \in \boldsymbol{E}\right\} .
$$

Clearly, every copy $O_{1}$ of $O$ defines the same diagram $\overline{O_{1}}$, which we denote simply by $\bar{O}$. Similarly, we define $\bar{W}$.

Example 3.21. If

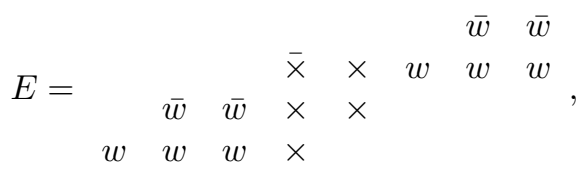

then the four cells labelled $\bar{w}$ denote two copies of $\bar{W}$, while the single $\bar{x}$ denotes $\bar{O}$. In general, however, $\bar{W}$ need not be a subset of $W$. For example, we can have

$$
E=\begin{array}{ccc} 
& \overline{\times} & \bar{w} \\
\bar{w} & \times & w \\
w & \times &
\end{array}
$$

where the cells on the top row comprise one copy of $\bar{W}$. Part of a second copy of $\bar{W}$ is also shown. 
Let us make some observations about Definition 3.20,

$\circ \overline{O_{1}}$ is nothing more than $\widetilde{O_{1}}$. However, using the notation $\widetilde{O}$ in what follows would have the potential to cause confusion, and using $\bar{O}$ instead will help to keep our notation consistent.

- We see that $\bar{O}$ and $\bar{W}$ are the shapes that result when we remove the infinite southeast ribbon from $\boldsymbol{E}$.

- Observe that $\bar{O}$ and $\bar{W}$ are skew diagrams and that neither one need be connected.

It turns out that we will need one further assumption about the structure of $E$. We conjecture below that this final assumption encompasses exactly what we need for our expression for $s_{D{ }_{W} E}$ to hold. In $\boldsymbol{E}=E^{\amalg_{W} \infty}$, Hypothesis 【 tells us that no two copies of $\bar{W}$ will be adjacent.

Hypothesis 3.22. Suppose that $E=W O W$. Assume that $E$ satisfies the following condition:

(IV) In $\boldsymbol{E}$, no copy of $\bar{O}$ is adjacent to a copy of $\bar{W}$.

Remark 3.23. Suppose we construct a second copy of $\boldsymbol{E}$ which is the translation of $\boldsymbol{E}$ one position northwest. Then $\bar{O}$ and $\bar{W}$ are exactly the shapes that form the overlap of the two copies of $\boldsymbol{E}$. Intuitively, Hypothesis 3.22 tells us that the overlap will be well-behaved: it will break up nicely into disjoint copies of $\bar{O}$ and $\bar{W}$.

In [14, $\bar{W}$ is always empty, so this hypothesis is not necessary.

The final construction required for our main results is a map on symmetric functions that will give an algebraic interpretation of the diagrammatic operation ${ }^{\circ} W$. We note that the definition below is the natural generalisation of [14, Definition 7.18].

Definition 3.24. Let $E$ and $W$ be skew diagrams such that $E=W O W$. Consider the map of sets

$$
\begin{array}{ccc}
\Lambda & \stackrel{(-) \circ_{W} s_{E}}{\longmapsto} & \Lambda \\
0 & \longmapsto & 0 \\
f & \longmapsto & f \circ_{W} s_{E}
\end{array}
$$

that consists of the composition of the following two maps $\Lambda \rightarrow \Lambda[t] \rightarrow \Lambda$ if $f \neq 0$. If we think of $\Lambda$ as the polynomial algebra $\mathbb{Z}\left[h_{1}, h_{2}, \ldots\right]$, then we can temporarily grade $\Lambda$ and $\Lambda[t]$ by setting $\operatorname{deg}(t)=\operatorname{deg}\left(h_{r}\right)=1$ for all $r$. The first map $\Lambda \rightarrow \Lambda[t]$ then homogenises a polynomial in the $h_{r}$ with respect to the above grading, using the variable $t$ as the homogenisation variable.

Meanwhile the second map is given by

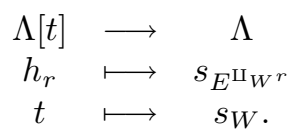

For example, if $f=h_{1} h_{2} h_{3}-\left(h_{3}\right)^{2}-h_{2} h_{4}+h_{6}$, then its image under the first map is $h_{1} h_{2} h_{3}-\left(h_{3}\right)^{2} t-h_{2} h_{4} t+h_{6} t^{2}$. Therefore,

$$
f \circ_{W} s_{E}=s_{E} s_{E^{\amalg} W^{2}} s_{E^{\amalg} W^{3}}-\left(s_{E^{\amalg} W^{3}}\right)^{2} s_{W}-s_{E^{\amalg} W^{2}} s_{E^{\amalg} W^{4}} s_{W}+s_{E^{\amalg} W^{6}}\left(s_{W}\right)^{2} \text {. }
$$

If $f=s_{D}$ for some skew diagram $D$, then we see that there is a nice way to think of $f \circ{ }_{W} s_{E}$ in terms of the Jacobi-Trudi decomposition matrix for $s_{D}$. Specifically, we homogenise by writing each entry of the form $s_{\emptyset}$ in the Jacobi-Trudi decomposition 
matrix as $h_{0}$. Then we replace $s_{(r)}=h_{r}$ by $E^{\amalg_{W} r}$ for $r \geq 0$. With the convention that $E^{\amalg_{W} 0}=W$, we now have that $s_{D} \circ_{W} s_{E}$ is simply the determinant of the resulting matrix. The reader is invited to check that the example above corresponds to this rule applied to the case of $f=s_{D}$ with $D=(4,2,2) /(1,1)$. Consequently, we have

Lemma 3.25. Let $\phi_{1}, \ldots, \phi_{r}$ be the ribbons of the Jacobi-Trudi decomposition of a skew diagram $D$. For skew diagrams $E$ and $W$ with $E=W O W$, we have

$$
s_{D} \circ_{W} s_{E}=\left(\operatorname{det}\left(s_{\left(\phi_{i} \# \phi_{j}\right)}\right)_{i, j=1}^{r} \circ_{W} s_{E}\right)=\operatorname{det}\left(s_{\left(\phi_{i} \# \phi_{j}\right)_{W} E}\right)_{i, j=1}^{r},
$$

where $\emptyset_{\circ_{W}} E$ is defined to be $W$, and where (undefined $\circ_{W} E$ ) is undefined.

Let $\widehat{D}$ denote the subset of elements of $D$ that have another element of $D$ one position to their south. Notice that $|\widehat{D}|=|\widetilde{D}|+r-1$, where $r$ is the number of rows in $D$. For symmetric functions $f$ and $g$, we will write $f= \pm g$ to mean that either $f=g$ or $f=-g$.

We are finally ready to put everything together and start reaping the rewards of our hard work.

Conjecture 3.26. For any skew diagram D, and a skew diagram $E$ satisfying Hypotheses [- IV] we have

$$
s_{D \circ_{W} E}\left(s_{\bar{W}}\right)^{|\widehat{D}|}\left(s_{\bar{O}}\right)^{|\widetilde{D}|}= \pm\left(s_{D} \circ_{W} s_{E}\right) .
$$

The sign on the right-hand side is a plus sign if $E=W \rightarrow O \rightarrow W$ or $E=W \uparrow$ $O \uparrow W$, and otherwise depends only on D. Furthermore, if $E$ does not satisfy Hypothesis IV, then there exists a skew diagram $D$ for which (3.2) fails to hold.

We can prove (3.2) when $E$ is of the form $W \rightarrow O \uparrow W$ or $W \uparrow O \rightarrow W$. However, our proof techniques require one further assumption for the two other forms of $E$.

Hypothesis 3.27. If $E=W \rightarrow O \rightarrow W$ or $E=W \uparrow O \uparrow W$, then we assume that:

(V) In $E$, at least one copy of $W$ has just one cell adjacent to $O$.

Theorem 3.28. For any skew diagram $D$, and a skew diagram $E$ satisfying $\mathrm{Hy}$ potheses I- - V, we have

$$
s_{D{ }_{W} E}\left(s_{\bar{W}}\right)^{|\widehat{D}|}\left(s_{\bar{O}}\right)^{|\widetilde{D}|}= \pm\left(s_{D} \circ_{W} s_{E}\right) .
$$

The sign on the right-hand side is a plus sign if $E=W \rightarrow O \rightarrow W$ or $E=W \uparrow O \uparrow$ $W$, and otherwise depends only on $D$.

Due to its length, we postpone the proof and devote the next section to it.

Remark 3.29. Again, we can compare what appears here with the relevant parts of 14. If $W$ is empty and either $D$ or $E$ is a ribbon, we obtain

$$
s_{D \circ_{\emptyset} E}=s_{D} \circ_{\emptyset} s_{E},
$$

which is their (7.2) and Proposition 7.5. If $D$ and $W$ are ribbons with $\bar{W}=\emptyset$, and $E=W \rightarrow O \rightarrow W$ or $E=W \uparrow O \uparrow W$, we obtain

$$
s_{D{ }_{W} E}=s_{D} \circ_{W} s_{E},
$$

which is equivalent to [14, Theorem 7.20]. In the case that $E$ is a ribbon of the form $E=W \rightarrow O \uparrow W$ or $E=W \uparrow O \rightarrow W$, we obtain a result that has no analogue in 14, but which implies the ribbon staircase equivalence in its Theorem 7.30. We will say more about this in Remark 3.33 . 
Remark 3.30. We can think of $\left(s_{\bar{W}}\right)^{|\widehat{D}|}\left(s_{\bar{O}}\right)^{|\widetilde{D}|}$ as the term introduced by the overlaps in $D \circ_{W} E$. This is consistent with (3.4) and (3.5), and with our observation that $\bar{W}$ and $\bar{O}$ are the shapes that form the overlap of $\boldsymbol{E}$ and a copy of $\boldsymbol{E}$ translated one position northwest.

While the major proof in this article is that of Theorem 3.28, our main target has been the following result, which serves as a mechanism for building skewequivalences.

Theorem 3.31. Suppose we have skew diagrams $D, D^{\prime}$ with $D \sim D^{\prime}$, and $E=$ WOW satisfying Hypotheses $\square-\mathrm{V}$. Then

$$
D^{\prime} \circ_{W} E \sim D \circ_{W} E \sim D \circ_{W^{*}} E^{*} .
$$

Proof. If $D \sim D^{\prime}$, then $s_{D}=s_{D^{\prime}}$ and so $s_{D}{ }^{\circ} W s_{E}=s_{D^{\prime}}{ }^{\circ} W s_{E}$. Since the left-hand side of (3.3) is Schur-positive, the sign on the right-hand side of (3.3) is determined by $s_{D}{ }^{\circ} W s_{E}$. By Theorem $3.3,|\widetilde{D}|=\left|\widetilde{D^{\prime}}\right|$ and $|\widehat{D}|=\left|\widehat{D^{\prime}}\right|$. Applying Theorem 3.28 then yields the first equivalence.

The second equivalence follows from the first and from Proposition 3.4 and Lemma 3.19)(iii) since

$$
D \circ_{W} E \sim\left(D \circ_{W} E\right)^{*}=D^{*} \circ_{W^{*}} E^{*} \sim D \circ_{W^{*}} E^{*} .
$$

Example 3.32. [14, Section 9] contains a list of the 6 skew-equivalences involving skew diagrams with at most 18 cells that are not explained by the results there. Using the equivalences of Theorem 3.31 we can now explain these equivalences. In all cases, let $D=\underset{\times}{\times} \times$ and $D^{\prime}=D^{*}$. Letting

$$
E=\times \begin{array}{lll} 
& w & w \\
w & \times &
\end{array}
$$

the first equivalence of (3.6) gives

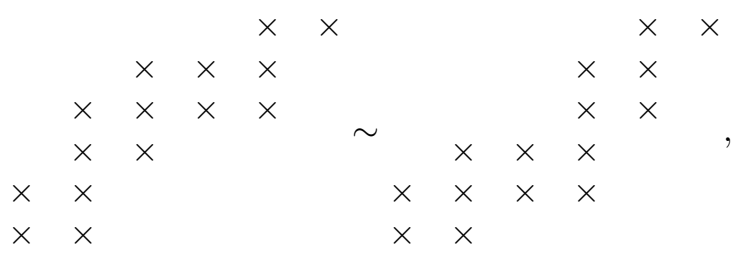

which is the first of the 6 equivalences. With

$$
E=\begin{array}{llll}
\times & \times & w & w \\
w & w & &
\end{array}
$$

we get the second equivalence, which is

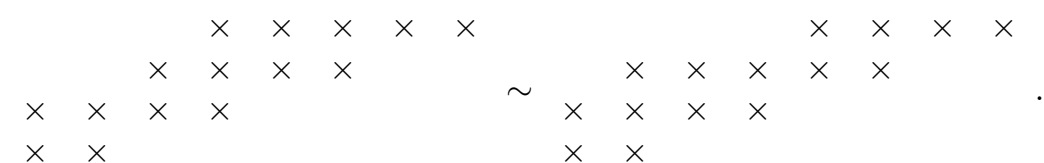


The remaining equivalences are obtained by setting $E$ to be

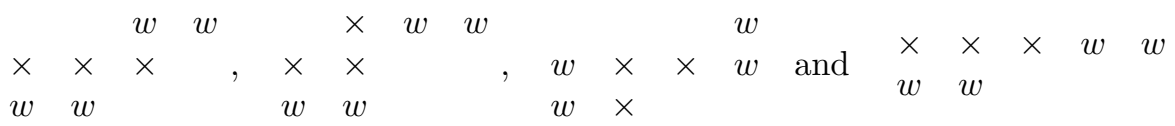

respectively.

Remark 3.33. The case when $E$ is a ribbon of the form $W \rightarrow O \uparrow W$ or $W \uparrow O \rightarrow W$ falls under the ribbon staircase construction of [14]. Theorem 7.30 there amounts to the equivalence $D^{*} \circ_{W} E \sim D \circ_{W} E$. Therefore, the first equivalence of (3.6), even when $E$ is just a ribbon, shows that more general ribbon staircase equivalences exist. As a first example, one could take $E=\underset{w}{\underset{w}{w}}$ and $D \sim D^{\prime}$ to be the unique non-trivial skew-equivalence for skew diagrams with at most 8 cells, i.e.

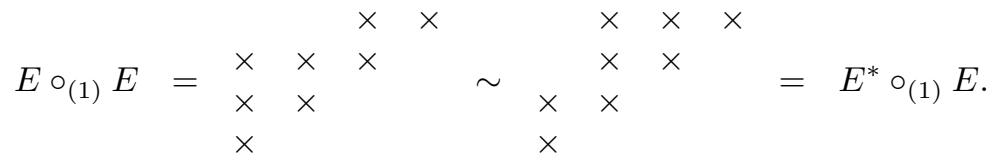

We leave the construction of $D \circ_{W} E$ and $D^{\prime} \circ_{W} E$, each of which has 20 cells, as an exercise.

\section{Proof of Theorem 3.28}

We devote this section to our main proof. As we will see, the proof divides into two cases: $E=W \rightarrow O \rightarrow W$ and $E=W \rightarrow O \uparrow W$. The first thing we will do is show that proofs of these two cases respectively imply the result for $E=W \uparrow O \uparrow W$ and $E=W \uparrow O \rightarrow W$. The proof of each case has two major steps. The first is to define an appropriate outside ribbon decomposition of $D{ }^{\circ} W E$. The second step is to apply Sylvester's Determinantal Identity to the resulting Hamel-Goulden matrix and to show that (3.3) results.

4.1. Reduction to two cases. If $W=\emptyset$, we are in the case $E=W \rightarrow 0 \rightarrow W$, which is one of the cases we will prove explicitly; therefore, assume that $W \neq \emptyset$. For the following reasons, we see that (3.3) holds for $D$ and $E$ if and only if the corresponding equation for $D^{*}$ and $E^{*}$ holds:

○ By Proposition 3.4 and Lemma 3.19 (ii), $s_{D_{{ }^{W}} E}=s_{D^{*} \circ_{W^{*}} E^{*}}$.

○ We have $E^{*}=W^{*} O^{*} W^{*}$, and we see that $\overline{O^{*}}=(\bar{O})^{*}$ and $\overline{W^{*}}=(\bar{W})^{*}$.

○ $|\widehat{D}|=\left|\widehat{D^{*}}\right|$ and $|\widetilde{D}|=\left|\widetilde{D^{*}}\right|$.

○ By Proposition 3.4 and since $\left(E^{\amalg_{W} n}\right)^{*}=\left(E^{*}\right)^{\amalg_{W * n}}, s_{D} \circ_{W} s_{E}=s_{D^{*}} \circ_{W}{ }^{*}$ $s_{E^{*}}$.

Moreover, applying the $\omega$ involution that sends $s_{D}$ to $s_{D^{t}}$, we get that (3.3) holds for $D$ and $E$ if and only if

$$
s_{\left(D \circ_{W} E\right)^{t}}\left(s_{(\bar{W})^{t}}\right)^{|\widehat{D}|}\left(s_{(\bar{O})^{t}}\right)^{|\widetilde{D}|}= \pm\left(s_{D} \circ_{W}{ }^{t} s_{E^{t}}\right) .
$$

Applying a similar argument to that for $D^{*}$ and $E^{*}$, we get that (4.1) holds if and only if

$$
s_{D^{*}{ }_{W^{t}} E^{t}}\left(s_{\overline{W^{t}}}\right)^{\mid \widehat{D^{*} \mid}}\left(s_{\overline{O^{t}}}\right)^{\mid \widetilde{D^{*} \mid}}= \pm\left(s_{D^{*}} \circ_{W^{t}} s_{E^{t}}\right),
$$

i.e. (3.3) holds for $D$ and $E$ if and only if the corresponding equation for $D^{*}$ and

$E^{t}$ holds. Therefore, (3.3) holds for $E$ and all skew diagrams $D$ if and only if it also 
holds with $E^{*}, E^{t}$ or $\left(E^{*}\right)^{t}$ in place of $E$, and all skew diagrams $D$. Furthermore, $E^{*}, E^{t}$ and $\left(E^{*}\right)^{t}$ satisfy Hypotheses $\square-\nabla$ if and only if $E$ does. Therefore, we can assume that $E$ is arranged in a convenient manner:

(1) If $E=W \rightarrow O \rightarrow W$ or $E=W \uparrow O \uparrow W$, then let us choose $E$ so that $E=W \rightarrow O \rightarrow W$ and $W_{s w}$ has at least as many rows in common with $O$ as $W_{n e}$. By Hypothesis $\mathbf{V}, W_{n e}$ thus has just one row in common with $O$.

(2) If $E=W \rightarrow O \uparrow W$ or $E=W \uparrow O \rightarrow W$, we will assume that $E=W \rightarrow O \uparrow$ $W$.

The advantage of these forms of $E$ is that every copy of $\bar{W}$ is contained in the corresponding copy of $W$.

Let us also reduce the second assertion in Theorem 3.28 to these two cases. Since the left-hand side of (3.3) is Schur-positive, the sign on the right-hand side depends only on $s_{D} \circ_{W} s_{E}$. Therefore, it follows from our argument above that the signs on the right-hand side in front of $s_{D}{ }^{\circ} W s_{E}$ and $s_{D^{*}}{ }^{\circ} W^{t} s_{E^{t}}$ will be the same. We know that if $E=W \uparrow O \uparrow W$, then $E^{t}=W^{t} \rightarrow O^{t} \rightarrow W^{t}$. Therefore, we get a plus sign on the right-hand side for every skew diagram $D$ and every $E=W \uparrow O \uparrow W$ satisfying Hypotheses $\square-\square$, if and only if the same applies when $E=W \rightarrow O \rightarrow W$ satisfying the hypotheses. It also follows from our argument above that the signs on the right-hand side in front of $s_{D^{\circ} W} s_{E}$ and $s_{D^{\circ}}{ }_{\left(W^{*}\right)^{t}} s_{\left(E^{*}\right)^{t}}$ will be the same. If $E=W \uparrow O \rightarrow W$, then $\left(E^{*}\right)^{t}=\left(W^{*}\right)^{t} \rightarrow\left(O^{*}\right)^{t} \uparrow\left(W^{*}\right)^{t}$. Hence, for fixed $D$, if the sign on the right-hand side of (3.3) is the same for all $E$ of the form $W \rightarrow O \uparrow W$ satisfying the hypotheses, then it will be the same for all $E$ of the forms $W \rightarrow O \uparrow W$ and $W \uparrow O \rightarrow W$ satisfying the hypotheses.

It will now be necessary to treat Cases 1 and 2 above separately.

4.2. The outside decomposition for Case 1, Our first major task is to construct an appropriate ribbon decomposition for $D \circ_{W} E$. For $d \in D$ and $e \in E$, let us write $d \circ_{W} e$ to denote the cell that corresponds to $e$ in the $E_{d}$ copy of $E$ in $D \circ_{W} E$. Then until further notice, for $D^{\prime} \subseteq D$ and $E^{\prime} \subseteq E$, we define $D^{\prime}{ }^{\circ}{ }_{W} E^{\prime}$ to be the subset of $D \circ_{W} E$ consisting of those cells of the form $d \circ_{W} e$, where $d \in D^{\prime}$ and $e \in E^{\prime}$. We construct a ribbon decomposition for $D \circ_{W} E$ by first constructing a ribbon decomposition for $D$. As mentioned in Remark 3.15, we will take the Jacobi-Trudi decomposition so that our ribbons are simply the rows $\phi_{1}, \ldots, \phi_{r}$ from north to south of $D$. Let $\Phi$ denote the cutting strip of this decomposition, which is just a single row of size $\left|n w_{D}\right|$. A ribbon decomposition for $\phi_{1} \circ_{W} E$ is now suggested by Definition 3.20, Indeed, every copy of $E$ can be partitioned into two copies of $\bar{W}$, one copy of $\bar{O}$ and a ribbon south $_{E}$ which runs along the south border of $E$. Extending this idea, $\phi_{1}{ }^{\circ} W E$ can be partitioned into $\left|\phi_{1}\right|+1$ copies of $\bar{W},\left|\phi_{1}\right|$ copies of $\bar{O}$ and a ribbon south $_{\phi_{1} \circ_{W} E}$ obtained by amalgamating the $\left|\phi_{1}\right|$ copies of south $_{E}$. Construct the southeast decompositions for the copies of $\bar{W}$ and $\bar{O}$ to obtain a ribbon decomposition for $\phi_{1}{ }^{\circ} W E$. For example, if

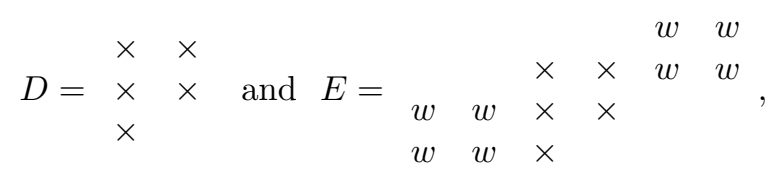

then $\phi_{1} \circ_{W} E$ has a ribbon decomposition given by the ribbons labelled 1, 8, 9, 10, 11, 12 in Figure 3. 


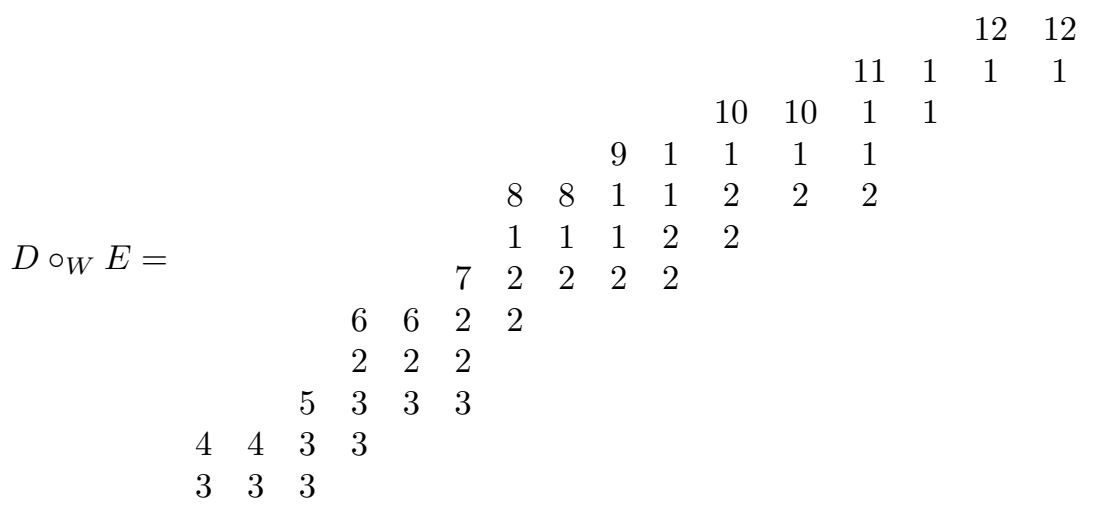

FiguRE 3

Now consider what happens when we add in the next part of $D \circ_{W} E$, namely $\phi_{2} \circ_{W} E$. Some of $\phi_{2}{ }^{\circ} W E$ will have already been included as elements of $\phi_{1} \circ_{W} E$, while some will be new. If $d^{\prime}$ is one position northwest of $d$ in $D$, then it follows from Definition 3.11 that $E_{d^{\prime}}$ will be one position northwest of $E_{d}$ in $D \circ_{W} E$. Furthermore, we see that the $\bar{O}$ and the two copies of $\bar{W}$ are exactly the subset of $E_{d}$ that will be contained in $E_{d^{\prime}}$. In particular, this implies that the ribbon $\phi_{2} \circ_{W}$ south $_{E}$ will be new. Also, for $d \in \phi_{2}$, the cells of the copy of $\bar{O}$ in $d \circ_{W} E$ will be new if and only if there is no cell $d^{\prime}$ one position northwest of $d$ in $D$, i.e. if and only if $d \in n w_{D}$. The same applies to the cells of the lower copy of $\bar{W}$ in $d \circ_{W} E$. The cells of the upper copy of $\bar{W}$ in $d \circ_{W} E$ will be new if and only if there is no cell $d^{\prime}$ one position north of $d$ in $D$. We conclude that $\phi_{2}{ }^{\circ} W E$ contributes $\left|\phi_{2} \cap n w_{D}\right|$ new copies of both $\bar{O}$ and $\bar{W}$ to $D \circ_{W} E$.

Continuing in this manner, $D \circ_{W} E$ can be partitioned into the ribbons $\phi_{i} \circ_{W}$ south $_{E}$ for $i=1, \ldots, r$, along with $\left|n w_{D}\right|$ copies of $\bar{O}$ and $\left|n w_{D}\right|+1$ copies of $\bar{W}$. Due to Hypotheses $\Pi$ and IV the copies of $\bar{O}$ and $\bar{W}$ are all pairwise non-adjacent. Using Hypothesis IV] one can also check that these copies of $\bar{O}$ and $\bar{W}$, as well as the endpoints of the ribbons $\phi_{i} \circ_{W}$ south $_{E}$, are on the outside of $D \circ_{W} E$ in the appropriate sense. Therefore, we can construct an outside ribbon decomposition $\theta_{1}, \ldots, \theta_{N}$ for $D \circ_{W} E$ as follows:

○ For $i=1, \ldots, r$, let $\theta_{i}:=\phi_{i} \circ_{W}$ south $_{E}$.

- Construct the southeast decomposition for each of the $\left|n w_{D}\right|+1$ copies of $\bar{W}$ and each of the $\left|n w_{D}\right|$ copies of $\bar{O}$. Label the resulting ribbons $\theta_{r+1}, \ldots, \theta_{N}$ from southwest to northeast according to their southwest endpoints.

We let $\Theta$ denote the cutting strip of this ribbon decomposition. Figure 3 is an example of such a decomposition.

4.3. Applying Sylvester's determinantal identity in Case 1, Now that our outside ribbon decomposition of $D \circ_{W} E$ is defined, we proceed to the second part of the proof for $E=W \rightarrow O \rightarrow W$. We wish to apply Sylvester's Determinantal Identity to $M$, the Hamel-Goulden matrix for our decomposition $\theta_{1}, \ldots, \theta_{N}$. We let the $S$ in Theorem 2.4 be the set $\{r+1, \ldots, N\}$. Then $M[S, S]$ is block lower-triangular, and the blocks on the diagonal alternate between Hamel-Goulden matrices for $\bar{W}$ 
and Hamel-Goulden matrices for $\bar{O}$. The left-hand side of (2.3) is thus

$$
s_{D{ }_{W} E}\left(\left(s_{\bar{W}}\right)^{\left|n w_{D}\right|+1}\left(s_{\bar{O}}\right)^{\left|n w_{D}\right|}\right)^{r-1} .
$$

We next wish to evaluate $M[S \cup\{i\}, S \cup\{j\}]$ for $i, j \in\{1,2, \ldots, r\}$. The topleft entry of this submatrix is $s_{\theta_{i} \# \theta_{j}}$. The remaining entries of the first row are $s_{\theta_{i} \# \theta_{l}}$ for $l=r+1, \ldots, N$. The remaining entries of the first column are $s_{\theta_{k} \# \theta_{j}}$ for $k=r+1, \ldots, N$. The $(k, l)$-entry for $k, l>1$ is $s_{\theta_{k+r-1} \# \theta_{l+r-1}}$. Therefore, our goal of evaluating $M[S \cup\{i\}, S \cup\{j\}]$ has the following two parts:

(a) If $\theta_{i} \# \theta_{j}$ is defined, then $s_{\theta_{i} \# \theta_{l}}=s_{\left(\theta_{i} \# \theta_{j}\right) \# \theta_{l}}$ and $s_{\theta_{k} \# \theta_{j}}=s_{\theta_{k} \#\left(\theta_{i} \# \theta_{j}\right)}$. Therefore, we wish to show that a skew diagram $F$ with the following property exists: when using the cutting strip $\Theta$, the ribbons of the resulting decomposition of $F$ correspond to the same portions of $\Theta$ as

$$
\theta_{i} \# \theta_{j}, \theta_{r+1}, \theta_{r+2}, \ldots, \theta_{N} .
$$

Then $M[S \cup\{i\}, S \cup\{j\}]$ for $i, j \in\{1,2, \ldots, r\}$ will be the Hamel-Goulden matrix for $F$.

(b) If $\theta_{i} \# \theta_{j}$ is undefined, then we wish to show that $\operatorname{det} M[S \cup\{i\}, S \cup\{j\}]=0$.

We first show (b). In our running example, we can take $i=3$ and $j=1$. We will show that, for some $t$, the submatrix of $M[S \cup\{i\}, S \cup\{j\}]$ consisting of its first $t+1$ rows has rank less than $t+1$, implying the result. As usual, let $p\left(\theta_{k}\right)$ and $q\left(\theta_{k}\right)$ respectively denote the starting contents and ending contents of the ribbon $\theta_{k}$. We know that $\theta_{k} \# \theta_{l}$ is defined if and only if $p\left(\theta_{l}\right) \leq q\left(\theta_{k}\right)+1$. We also know that $q\left(\theta_{i}\right)$ is the content of the northeast endpoint $q_{i}$ of the ribbon $\phi_{i}{ }^{\circ} W$ south ${ }_{E}$. In particular, $q_{i}$ is in a copy $W_{i}$ of $W$. We will be comparing the positions of copies of $W, \bar{W}$ and $\bar{O}$ according to how far southwest or northeast they are. We will say that a subdiagram $A$ of $D \circ_{W} E$ is weakly southwest of a subdiagram $B$ if the maximum content of the cells of $A$ is weakly less than the maximum content of the cells of $B$. As a variation of this, we will say that $A$ is strictly northeast of $B$ if the minimum content in $A$ is strictly greater than the maximum content in $B$. In effect, we are comparing subdiagrams according to their positions on the cutting strip of $D \circ_{W} E$. Let $t$ be the maximum positive integer such that $\theta_{r+t}$ is in the southeast decomposition of a copy of $\bar{W}$ or $\bar{O}$ that is weakly southwest of $W_{i}$, or set $t=0$ if no such positive integer exists. In our running example where $i=3$, we have that $r+t=6$. Due to the way we have labelled $\theta_{r+1}, \ldots, \theta_{N}$, this definition of $t$ tells us that $\theta_{r+1}, \ldots, \theta_{r+t}$ are contained in copies of $\bar{W}$ and $\bar{O}$ weakly southwest of $W_{i}$, while $\theta_{r+t+1}, \ldots, \theta_{N}$ are contained in copies strictly northeast of $W_{i}$. We have that:

○ $p\left(\theta_{j}\right)>q\left(\theta_{i}\right)+1$, since $\theta_{i} \# \theta_{j}$ is undefined.

○ $p\left(\theta_{l}\right)>q\left(\theta_{i}\right)+1$ for $l=r+t+1, \ldots, N$. Indeed, suppose $p\left(\theta_{l}\right) \leq q\left(\theta_{i}\right)+1$, and let $p_{l}$ denote the southwest endpoint of $\theta_{l}$. By Hypothesis II the only danger is if $\theta_{l}$ is contained in the copy $O_{l}$ of $O$ that is immediately northeast of $W_{i}$. Since $\theta_{i} \# \theta_{j}$ is undefined, we know that $i \neq 1$, and we also know by definition of $q_{i}$ that $W_{i}$ is the northeasternmost copy of $W$ in $\phi_{i}{ }^{\circ} W E$. Therefore, there exists a copy $W_{l}$ of $W$ that is a translation of $W_{i}$ one position to the northwest. Observe that $W_{l}$ and $O_{l}$ are from the same copy of $E$, and let $q_{l} \in W_{l}$ denote the translation of $q_{i}$ one position to 
the northwest. Since $q_{i}$ has no cell immediately to its east, we know that $q_{l}$ has a cell $o$ of $O_{l}$ one position to its east. In fact, we know that $o \notin \overline{O_{l}}$, so, in particular, $o \neq p_{l}$. Since $p\left(\theta_{l}\right) \leq q\left(\theta_{i}\right)+1, p_{l}$ must be strictly west and weakly north of $o$. However, since $o, p_{l} \in O_{l}$ and $O_{l}$ is a skew diagram, this implies that $q_{l}$ is contained in $O_{l}$, a contradiction.

$\circ p\left(\theta_{j}\right)>q\left(\theta_{k}\right)+1$, for $k=r+1, \ldots, r+t$. Indeed, we know that $p\left(\theta_{j}\right)$ is the content of the southwest endpoint $p_{j}$ of the ribbon $\phi_{j}{ }^{\circ} W$ south $_{E}$. Therefore, $p_{j}$ is an element of a copy $W_{j}$ of $W$. However, since $p\left(\theta_{j}\right)>q\left(\theta_{i}\right)+1$, $W_{j}$ must be strictly northeast of $W_{i}$, while $\theta_{k}$ is weakly southwest of $W_{i}$. Hypothesis \I then implies the claim.

○ $p\left(\theta_{l}\right)>q\left(\theta_{k}\right)+1$, for $l=r+t+1, \ldots, N$ and $k=r+1, \ldots, r+t$. Indeed, $\theta_{l}$ is contained in a copy of $\bar{W}$ or $\bar{O}$ that is strictly northeast of $W_{i}$, while $\theta_{k}$ is contained in a copy of $\bar{W}$ or $\bar{O}$ that is weakly southwest of $W_{i}$. By Hypotheses II and IV no two copies of $\bar{W}$ or $\bar{O}$ are adjacent, implying the claim.

Therefore, $\theta_{k} \# \theta_{l}$ is undefined, and so $s_{\theta_{k} \# \theta_{l}}=0$, whenever $k \in\{i, r+1, r+$ $2, \ldots, r+t\}$ and $l \in\{j, r+t+1, r+t+2, \ldots, N\}$. This shows that the submatrix of $M[S \cup\{i\}, S \cup\{j\}]$ consisting of its first $t+1$ rows has rank at most $t$, as required for $(b)$.

To construct the diagram $F$ needed to establish (a), we move from the setting of $D \circ_{W} E$ to the setting of $\Phi \circ_{W} E$, recalling that $\Phi$ is the cutting strip for the JacobiTrudi decomposition of $D$, i.e. $\Phi$ is a single row of size $\left|n w_{D}\right|$. As a result, $d \circ_{W} e$ now denotes the cell that corresponds to $e$ in the $E_{d}$ copy of $E$ in $\Phi{ }^{\circ} E$. Subsequently, a term of the form $\Phi^{\prime} \circ_{W} E^{\prime}$ with $\Phi^{\prime} \subseteq \Phi$ and $E^{\prime} \subseteq E$ denotes those $d \circ_{W} e \in \Phi \circ_{W} E$ such that $d \in \Phi^{\prime}$ and $e \in E^{\prime}$. We will continue to use $\Theta$ as our cutting strip. Notice that this change does not affect the definition $\theta_{i}:=\phi_{i}{ }^{\circ} W$ south $_{E}$, since we get the same portion of $\Theta$ in the setting of $\Phi \circ_{W} E$ as we did in the $D \circ_{W} E$ setting. We have that

$$
\theta_{i} \# \theta_{j}=\left(\phi_{i} \circ_{W} \text { south }_{E}\right) \#\left(\phi_{j} \circ_{W} \text { south }_{E}\right)=\left(\phi_{i} \# \phi_{j}\right) \circ_{W} \text { south }_{E} \text {. }
$$

If $\phi_{i} \# \phi_{j}=\emptyset$, then $\left(\phi_{i} \# \phi_{j}\right) \circ_{W}$ south $_{E}$ should be defined to be the portion of $W$ that is contained in south ${ }_{E}$, namely $W \backslash \bar{W}$.

As usual, let $\Phi\left[p\left(\phi_{j}\right), q\left(\phi_{i}\right)\right]$ denote the portion of the cutting strip $\Phi$ for $D$ corresponding to $\phi_{i} \# \phi_{j}$. We will let the southwesternmost cell of the cutting strip have content 1 , so the cutting strip itself can be expressed as $\Phi\left[1,\left|n w_{D}\right|\right]$. Let $\bar{E}$ denote $E \backslash$ south $_{E}$, which we know in our case consists of two copies of $\bar{W}$ and a copy of $\bar{O}$. We write $\overline{W O}$ (resp. $\overline{O W}$ ) to denote the result of deleting the upper (resp. lower) copy of $\bar{W}$ from $\bar{E}$. When $\Phi\left[p\left(\phi_{j}\right), q\left(\phi_{i}\right)\right]$ is defined, we claim that the required skew diagram $F$ is the subset of $\Phi{ }^{\circ} W E$ consisting of

$$
\left(\Phi\left[p\left(\phi_{j}\right), q\left(\phi_{i}\right)\right]{ }_{W} \text { south }_{E}\right) \cup\left(\Phi \circ_{W} \bar{E}\right)
$$

In our running example, we could take $i=2$ and $j=3$. Then $F$ is the following skew diagram, where we label the cells according to the label of the corresponding 
cell in the ribbon decomposition of $D \circ_{W} E$ :

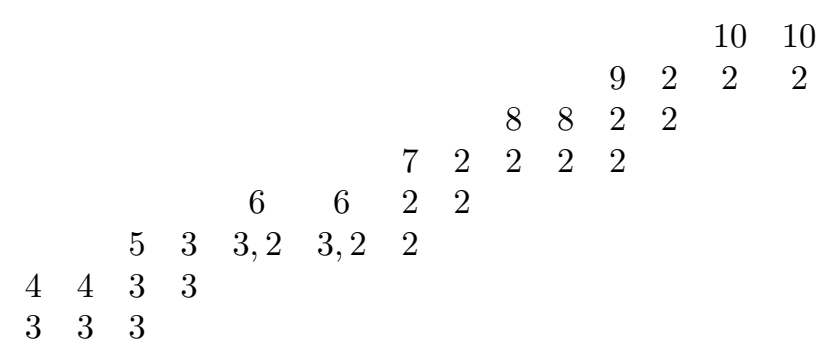

Intuitively, we have deleted the ribbon in $D \circ_{W} E$ labelled 1, and then translated some of the remaining ribbons to the southeast. Since translations to the southeast do not affect the contents of cells, for each remaining ribbon we are using the same portions of the cutting strip as before. The union of (4.3) is a disjoint union, since $\Phi$ is a ribbon and south $_{E}$ is disjoint from $\bar{E}$ in $E$. It can be rewritten as

$$
\begin{aligned}
& \left(\Phi\left[p\left(\phi_{j}\right), q\left(\phi_{i}\right)\right] \circ_{W} \text { south }_{E}\right) \sqcup\left(\Phi\left[1, p\left(\phi_{j}\right)-1\right] \circ_{W} \overline{W O}\right) \\
& \sqcup\left(\Phi\left[p\left(\phi_{j}\right), q\left(\phi_{i}\right)\right] \circ_{W} \bar{E}\right) \sqcup\left(\Phi\left[q\left(\phi_{i}\right)+1,\left|n w_{D}\right|\right] \circ_{W} \overline{O W}\right) \\
= & \left(\Phi\left[1, p\left(\phi_{j}\right)-1\right] \circ_{W} \overline{W O}\right) \sqcup\left(\Phi\left[p\left(\phi_{j}\right), q\left(\phi_{i}\right)\right] \circ_{W} E\right) \\
& \sqcup\left(\Phi\left[q\left(\phi_{i}\right)+1,\left|n w_{D}\right|\right] \circ_{W} \overline{O W}\right) .
\end{aligned}
$$

We make three observations about the latter disjoint union:

- The three terms are (not necessarily connected) skew diagrams when considered individually.

- Their union is obtained from $\Phi \circ_{W} E$ by removing an initial portion and a final portion of the south border ribbon $\Phi \circ_{W}$ south $_{E}$.

- Using Hypotheses [V] and [V] we see that at least one empty diagonal separates each pair of terms in the disjoint union.

Taken together, these observations tell us that the disjoint union is a skew diagram. By construction (see (4.3)), using the cutting strip $\Theta$, the ribbons of the resulting decomposition correspond to the same portions of $\Theta$ as $\theta_{i} \# \theta_{j}, \theta_{r+1}, \theta_{r+2}, \ldots, \theta_{N}$. Thus we have shown (回).

Evaluating the corresponding skew Schur function, we finally deduce that

$$
\operatorname{det} M[S \cup\{i\}, S \cup\{j\}]=s_{\left(\phi_{i} \# \phi_{j}\right) \circ_{W} E}\left(s_{\bar{W}} s_{\bar{O}}\right)^{\left|n w_{D}\right|-q\left(\phi_{i}\right)+p\left(\phi_{j}\right)-1},
$$

since $s_{\overline{O W}}=s_{\overline{W O}}=s_{\bar{W}} s_{\bar{O}}$.

Therefore, by Lemma 3.25, $\operatorname{det} \operatorname{Syl}(M, S)$ evaluates to

$$
\left(s_{\bar{W}} s_{\bar{O}}\right)^{I} \operatorname{det}\left(s_{\left(\phi_{i} \# \phi_{j} \circ_{W} E\right.}\right)_{i, j=1}^{r}=\left(s_{\bar{W}} s_{\bar{O}}\right)^{I}\left(s_{D} \circ_{W} s_{E}\right),
$$

where

$$
I=r\left(\left|n w_{D}\right|-1\right)-\sum_{i=1}^{r} q\left(\phi_{i}\right)+\sum_{j=1}^{r} p\left(\phi_{j}\right) .
$$

Plugging this and (4.2) into (2.3), we obtain

$$
s_{D \circ W}\left(s_{\bar{W}}\right)^{\left(\left|n w_{D}\right|+1\right)(r-1)-I}\left(s_{\bar{O}}\right)^{\left|n w_{D}\right|(r-1)-I}=s_{D} \circ_{W} s_{E} .
$$


Since

$$
\left|n w_{D}\right|(r-1)-I=-\left|n w_{D}\right|+\sum_{i=1}^{r}\left(q\left(\phi_{i}\right)-p\left(\phi_{i}\right)+1\right)=|D|-\left|n w_{D}\right|=|\widetilde{D}|
$$

and

$$
\left(\left|n w_{D}\right|+1\right)(r-1)-I=|\widetilde{D}|+r-1=|\widehat{D}|,
$$

we conclude that

$$
s_{D \circ_{W} E}\left(s_{\bar{W}}\right)^{|\widehat{D}|}\left(s_{\bar{O}}\right)^{|\widetilde{D}|}=s_{D} \circ_{W} s_{E},
$$

as required.

4.4. The outside decomposition for Case 2. Throughout, it will be advantageous to think of $D \circ_{E} W$ in terms of Definition 3.16. In particular, we know that when $d$ is one position south of $d^{\prime}$ on the same ribbon in $D$, then $E_{d}$ and $E_{d^{\prime}}$ appear in the form $E_{d} \amalg_{W} E_{d^{\prime}}$. It is necessary to explicitly add an extra copy of $W$ one position southeast of $E_{d} \cap E_{d^{\prime}}$ if and only if both $d$ and $d^{\prime}$ are elements of $s e_{D}$. Accounting for these extra copies of $W$ is what makes the notation of this case difficult. In Case 1, we wrote $d \circ_{W} e$ to denote the cell that corresponds to $e$ in the $E_{d}$ copy of $E$ in $D \circ_{W} E$. However, when $E=W \rightarrow O \uparrow W$, the cells of the extra copies of $W$ do not naturally take the form $d \circ_{W} e$ for any $d$ and $e$. We begin by concocting a way to rectify this situation.

Recall that $\emptyset \circ_{W} E=W$. So we could think of these extra copies of $W$ as being the contribution from some empty ribbons that we add to the northwest decomposition of $D$. Specifically, if $d$ is one position south of $d^{\prime}$ on the same ribbon in $D$ and $d, d^{\prime} \in s e_{D}$, then we can add imaginary cells $i(d)$ and $i\left(d^{\prime}\right)$ one position southeast of $d$ and $d^{\prime}$ respectively. Then to the northwest decomposition we add an empty ribbon which starts at $i\left(d^{\prime}\right)$ and ends at $i(d)$. When we have added the empty ribbons for all such $d$ and $d^{\prime}$, we call the resulting ribbon decomposition the enhanced northwest decomposition. The big advantage of this ribbon decomposition is that every ribbon of size $l$ in the enhanced northwest decomposition contributes $E^{\amalg_{W} l}$ to $D \circ_{W} E$, where we define $E^{\amalg_{W} 0}$ to be $W$ and where $D \circ_{W} E$ is exactly the union of these contributions, suitably positioned. For $D$ in Example 3.17, we would have

$$
\begin{array}{lcc} 
& a_{5} & \\
a_{3} & a_{4} & i\left(a_{5}\right) \\
a_{2} & b & i\left(a_{4}\right) . \\
a_{1} & i\left(a_{2}\right) & \\
& i\left(a_{1}\right) &
\end{array}
$$

Let $p\left(\phi_{i}\right)$ and $q\left(\phi_{i}\right)$ respectively denote the contents of the southwest and northeast endpoints of a ribbon $\phi_{i}$. We leave it as a nice exercise for the reader to check that the enhanced ribbon decomposition of any $D$ has $r$ ribbons, where $r$ is the number of rows of $D$. Therefore, let $\phi_{1}, \ldots, \phi_{r}$ denote this set of ribbons, ordering the ribbons so that $q\left(\phi_{1}\right) \geq q\left(\phi_{2}\right) \geq \cdots \geq q\left(\phi_{r}\right)$. In particular, we will have $\phi_{1}=n w_{D}$.

We are now in a good position to describe a ribbon decomposition for $D \circ_{W} E$. First let us give an example to which the reader can refer for intuition. Suppose 
that

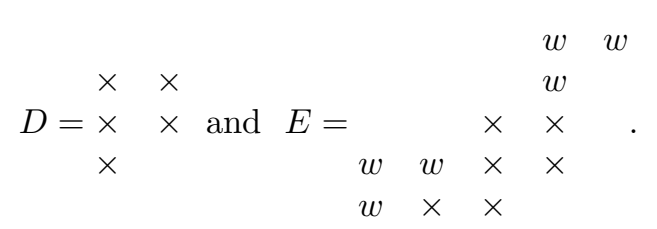

The ribbon decomposition of $D \circ_{W} E$ that we will construct is

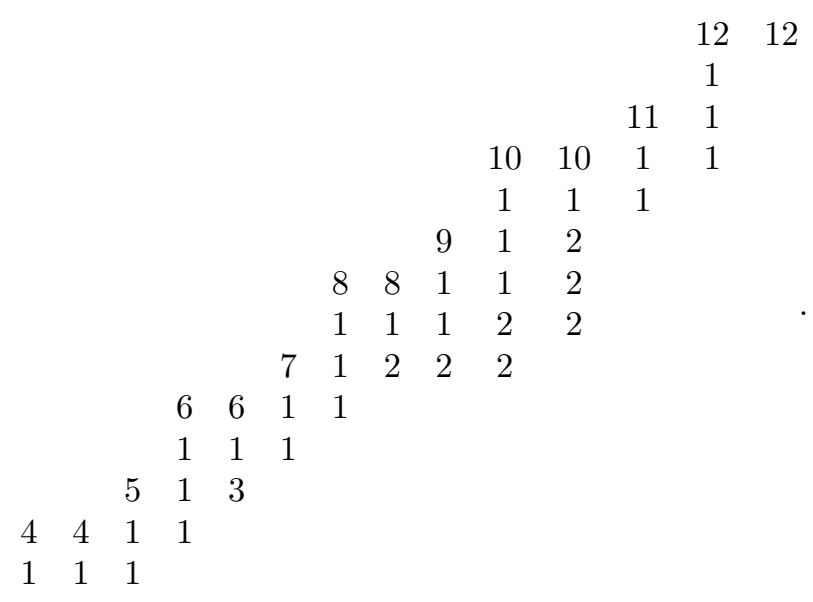

We must give a precise description of the general form of this ribbon decomposition. We will divide $D \circ_{W} E$ into two subdiagrams, $L$ and $U$, each of which is relatively easy to handle. We will then give $L$ a northwest decomposition and $U$ a decomposition that is close to a southeast decomposition. These two decompositions will be compatible, resulting in a global outside ribbon decomposition for $D \circ_{W} E$.

The upper subdiagram $U$ is the contribution from $n w_{D}$. In other words, $U$ is the unique subdiagram of shape $E^{\amalg\left|n w_{D}\right|}$ in $D \circ_{W} E$ that includes $n w_{D \circ_{W} E}$. In our example, $U$ is the subdiagram consisting of those cells with labels from the set $\{1,4,5,6, \ldots, 12\}$.

Now consider the contribution to $D \circ_{W} E$ from those ribbons of the enhanced northwest decomposition of $D$, other than $n w_{D}$. If $\phi_{i}$ has size $l$, then we noted that $\phi_{i}$ contributes the skew diagram $E^{\amalg_{W} l}$ to $D \circ_{W} E$. However, much of this contribution will be contained in the contribution of $\phi_{j}$, where $\phi_{j}$ is immediately to the northwest of $\phi_{i}$. As before, define south $_{E}$ as the result of removing the two copies of $\bar{W}$ and the copy of $\bar{O}$ from $E$.

For $i=1, \ldots, r$, in the skew diagram $E^{\amalg_{W} l}$ contributed by $\phi_{i}$, let $\phi_{i} \circ_{W}$ south $_{E}$ denote the subdiagram obtained by considering only south s $_{E}$ in each copy of $E$. For example, $\phi_{1} \circ_{W}$ south $_{E}=n w_{D} \circ_{W}$ south $_{E}$ is the ribbon labelled 1 in our example. It is clear that $\phi_{i}{ }^{\circ} W$ south $_{E}$ is a ribbon for $i=1, \ldots, r$. We can also see that $\phi_{1} \circ_{W}$ south $_{E}, \ldots, \phi_{r} \circ_{W}$ south $_{E}$ are disjoint in $D \circ_{W} E$, in part because $\phi_{1}, \ldots, \phi_{r}$ are disjoint in $D$. Furthermore, if $i>1$, then $\phi_{i}{ }^{\circ} W$ south $_{E}$ is exactly the contribution from $\phi_{i}$ that is not contained in the contribution of $\phi_{j}$, where $\phi_{j}$ is immediately northwest of $\phi_{i}$ in $D$. We let the lower subdiagram $L$ be the union of $\phi_{1} \circ_{W}$ south $_{E}, \ldots, \phi_{r} \circ_{W}$ south $_{E}$. One could think of $L$ as $D \circ_{W}$ south $_{E}$. In our example, $L$ is the subdiagram with cells labelled 1, 2, 3 . 
In our example, $U \cap L$ is the ribbon labelled 1. In general, we see that $U \cap L$ is the ribbon $\phi_{1} \circ_{W}$ south $_{E}$. We also have that $U \cup L=D \circ_{W} E$. Indeed, the empty ribbons from the enhanced northwest decomposition contribute the cells that come from the extra copies of $W$. If $\phi_{i}$ is empty, by definition $\phi_{i}{ }^{\circ} W s{ } t_{h}$ is going to be the set of cells of $W$ that are in south $_{E}$, i.e. $W \backslash \bar{W}$. We can now see that if a cell of $D \circ_{W} E$ is not in $\phi_{i} \circ_{W} \operatorname{south}_{E}$ for some $i$, then it must be an element of $E_{d}$ for some $d \in n w_{D}$, and hence is in $U$.

We can now define the ribbon decomposition $\theta_{1}, \ldots, \theta_{N}$ of $D \circ_{W} E$. For $i=$ $1, \ldots, r$, we let $\theta_{i}=\phi_{i} \circ_{W}$ south $_{E}$. Then, by definition of $L, \theta_{1}, \ldots, \theta_{r}$ give a ribbon decomposition of $L$. Certainly, if $\phi_{i}$ is empty, then $\theta_{i}$ is an outside ribbon in $L$. If $\phi_{i}$ is non-empty, then $\theta_{i}$ will be an outside ribbon in $L$ because $\phi_{i}$ was an outside ribbon in $D$. Furthermore, since $\theta_{1}$ is $n w_{L}$, it must be the cutting strip of this outside decomposition of $L$, and so $\theta_{1}, \ldots, \theta_{r}$ give the northwest ribbon decomposition of $L$. One can also check that $\theta_{1}, \ldots, \theta_{r}$ will be outside ribbons in $D \circ_{W} E$.

We proceed to decompose $U$ into ribbons in such a way that we get a global decomposition of $D \circ_{W} E$. The intersection of $U$ with $L$ is already the ribbon $\theta_{1}$, which includes all the cells of south $_{E}$ for the copies of $E$ that make up $U$. Therefore, all that remains of $U$ is $\left|n w_{D}\right|$ copies of $\bar{O}$ and $\left|n w_{D}\right|+1$ copies of $\bar{W}$. By Hypotheses $\amalg$ and IV these copies of $\bar{W}$ and $\bar{O}$ are pairwise non-adjacent. Construct the southeast decomposition for each copy of $\bar{W}$ and $\bar{O}$. Label the new ribbons $\theta_{r+1}, \ldots, \theta_{N}$ from southwest to northeast, according to their southwest endpoints. Then $\theta_{1}, \theta_{r+1}, \theta_{r+2}, \ldots, \theta_{N}$ is an outside ribbon decomposition for $U$. Therefore, we finally have an outside ribbon decomposition $\theta_{1}, \ldots, \theta_{N}$ for $D \circ_{W} E$. Let $\Theta$ denote the cutting strip of this decomposition.

4.5. Applying Sylvester's determinantal identity in Case 2, With the ribbon decomposition defined in the previous subsection, we now move to the second part of the proof of the case when $E=W \rightarrow O \uparrow W$. Exactly as in Case1 we apply Theorem 2.4 letting $M$ be the Hamel-Goulden matrix for the ribbon decomposition $\theta_{1}, \ldots, \theta_{N}$ and $S=\{r+1, \ldots, N\}$. For exactly the same reason as before, the left-hand side of (2.3) becomes

$$
s_{D \circ_{W} E}\left(\left(s_{\bar{W}}\right)^{\left|n w_{D}\right|+1}\left(s_{\bar{O}}\right)^{\left|n w_{D}\right|}\right)^{r-1} .
$$

We next wish to evaluate $M[S \cup\{i\}, S \cup\{j\}]$ for $i, j \in\{1,2, \ldots, r\}$. The topleft entry of this submatrix is $s_{\theta_{i} \# \theta_{j}}$. Again, we must first show that if $\theta_{i} \# \theta_{j}$ is undefined, then $\operatorname{det} M[S \cup\{i\}, S \cup\{j\}]=0$. We omit the proof of this since it is identical to the proof for Case 1 .

We next wish to show that a skew diagram $F$ with the following property exists: when using the cutting strip $\Theta$, the ribbons of the resulting decomposition of $F$ correspond to the same portions of $\Theta$ as $\theta_{i} \# \theta_{j}, \theta_{r+1}, \theta_{r+2}, \ldots, \theta_{N}$. Then $M[S \cup\{i\}, S \cup\{j\}]$ for $i, j \in\{1,2, \ldots, r\}$ will be the Hamel-Goulden matrix for $F$.

Since $\theta_{i} \# \theta_{j}$ is contained in $\theta_{1}$, and $\theta_{1}, \theta_{r+1}, \theta_{r+2}, \ldots, \theta_{N}$ are the ribbon decomposition for $U$, we wish to obtain $F$ as a subset of $U$. Our candidate $F$ is much simpler to define than in Case 1 in $U$, just replace $\theta_{1}$ with the subribbon of $\theta_{1}$ corresponding to $\theta_{i} \# \theta_{j}$. In our running example, taking $i=2$ and $j=3$ gives the 
skew diagram

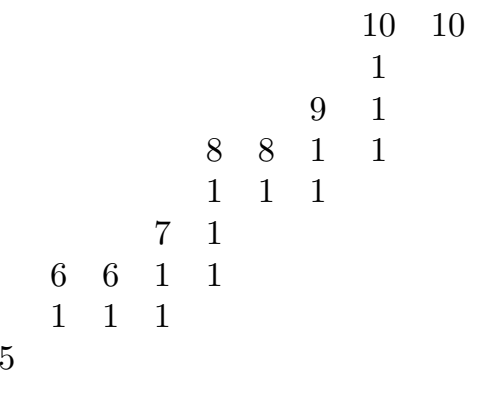

Define $\bar{E}, \overline{W O}$ and $\overline{O W}$ as in Case 1 . Writing $\Phi$ for $\phi_{1}$, the cutting strip of the northwest decomposition of $D$, we know that the contribution of $\Phi$ to $D \circ_{W} E$ is $U=E^{\amalg_{W}\left|n w_{D}\right|}$. If $\Phi^{\prime}$ is a portion of $\Phi$ and $E^{\prime} \subseteq E$, we will let $\Phi^{\prime} \circ_{W} E^{\prime}$ denote the subset of $U$ corresponding to $\Phi^{\prime}$ and $E^{\prime}$ in the natural way. Our candidate $F$ can thus also be written as

$$
\left(\Phi\left[p\left(\phi_{j}\right), q\left(\phi_{i}\right)\right] \circ_{W} \text { south }_{E}\right) \cup\left(\Phi \circ_{W} \bar{E}\right) .
$$

This is identical to (4.3). Proceeding exactly as in Case 1. we deduce that

$$
\operatorname{det} M[S \cup\{i\}, S \cup\{j\}]=s_{\left(\phi_{i} \# \phi_{j}\right) \circ_{W} E}\left(s_{\bar{W}} s_{\bar{O}}\right)^{\left|n w_{D}\right|-q\left(\phi_{i}\right)+p\left(\phi_{j}\right)-1} .
$$

At this point, we diverge from the proof of Case 1. We still, however, wish to plug our calculations into (2.3). In $\operatorname{det} \operatorname{Syl}(M, S)$, we can factor out the powers of $s_{\bar{W}} s_{\bar{O}}$ and divide the result into (4.5) as before, to obtain

$$
s_{D \circ_{W} E}\left(s_{\bar{W}}\right)^{|\widehat{D}|}\left(s_{\bar{O}}\right)^{|\widetilde{D}|}=\operatorname{det} H,
$$

where

$$
H=\left(s_{\left(\phi_{i} \# \phi_{j}\right){ }_{W} E}\right)_{i, j=1}^{r} .
$$

The skew diagram of the $(i, j)$-entry of $H$ is $\left(\phi_{i} \# \phi_{j}\right) \circ_{W} E$, which equals

$$
E^{\amalg_{W}\left(q\left(\phi_{i}\right)-p\left(\phi_{j}\right)+1\right)} .
$$

Recall that the ribbons $\phi_{1}, \ldots, \phi_{r}$ are those of the enhanced northwest decomposition of $D$, labelled so that $q\left(\phi_{1}\right) \geq q\left(\phi_{2}\right) \geq \cdots \geq q\left(\phi_{r}\right)$. We wish to relate these ribbons to the rows of $D$. In fact, since we know that $\operatorname{det} H \neq 0$, we have $q\left(\phi_{1}\right)>q\left(\phi_{2}\right)>\cdots>q\left(\phi_{r}\right)$. However, we certainly need not have $p\left(\phi_{1}\right)>p\left(\phi_{2}\right)>\cdots>p\left(\phi_{r}\right)$. To solve this problem, apply a permutation $\sigma^{-1}$ to the columns of $H$ to obtain a matrix $H^{\prime}$. The permutation $\sigma$ is chosen so that $p\left(\phi_{\sigma(1)}\right)>p\left(\phi_{\sigma(2)}\right)>\cdots>p\left(\phi_{\sigma(r)}\right)$. Then the skew diagram of the $(i, j)$-entry of $H^{\prime}$ is

$$
E^{\amalg_{W}\left(q\left(\phi_{i}\right)-p\left(\phi_{\sigma(j)}\right)+1\right)} .
$$

We will have $\operatorname{det} H=(-1)^{\operatorname{inv}(\sigma)} \operatorname{det} H^{\prime}$, where $\operatorname{inv}(\sigma)$ denotes the number of inversions of $\sigma$, explaining the appearance of the \pm sign in Theorem 3.28. This sign is completely determined by the enhanced northwest decomposition of $D$, and so will be the same for any $E$ of the form $W \rightarrow O \uparrow W$. 
To interpret $q\left(\phi_{i}\right)-p\left(\phi_{j}\right)+1$, we need to examine the enhanced northwest decomposition of $D$. The reader may wish to refer to (4.4). Clearly, the content of the northeasternmost cell of $D$ is $q\left(\phi_{1}\right)$. Now suppose $d$ is one position south of $d^{\prime}$ on $s e_{D}$. If $d$ and $d^{\prime}$ are on different ribbons of the northwest decomposition of $D$, then $d$ is clearly the northeast endpoint of $\phi_{i}$ for some $i$. Otherwise $d$ and $d^{\prime}$ are on the same ribbon of the northwest decomposition, in which case the content of $d$ is $q\left(\phi_{i}\right)$ for some empty ribbon $\phi_{i}$. Therefore, the content of the easternmost cell on every row of $D$ equals $q\left(\phi_{i}\right)$ for some $i$. Hence, for $i=1, \ldots, r, q\left(\phi_{i}\right)$ must be the content of the easternmost cell in the $i$ th row of $D$. If we write $D=\lambda / \mu$, with $\lambda$ chosen so that $\lambda_{1}$ and the length of $\lambda$ are minimal, then $q\left(\phi_{i}\right)=\lambda_{i}-i$.

Similarly, the content of the southwesternmost cell of $D$ is clearly $p\left(\phi_{\sigma(r)}\right)$. If $d$ is one position south of $d^{\prime}$ on $n w_{D}$, then $d$ and $d^{\prime}$ are obviously on the same ribbon, and hence every cell on $d$ 's diagonal goes north, as defined in Subsection 2.3. This means that the cells $e$ and $e^{\prime}$ on $s e_{D}$ with the same contents as $d$ and $d^{\prime}$ respectively satisfy either:

- $e$ appears one position south of $e^{\prime}$ on the same ribbon of the northwest decomposition; or

$\circ e^{\prime}$ is the southwest endpoint of a ribbon of the northwest decomposition of $D$.

In either of the two cases, the content of $e^{\prime}$, and hence the content of $d^{\prime}$, equals $p\left(\phi_{\sigma(j)}\right)$ for some $j=1, \ldots, r-1$. Since there are $r-1$ such $d^{\prime}$, we conclude that, for $j=1, \ldots, r, p\left(\phi_{\sigma(j)}\right)$ is the content of the westernmost cell in the $j$ th row of $D$, i.e. $p\left(\phi_{\sigma(j)}\right)=\mu_{j}+1-j$.

Therefore, $q\left(\phi_{i}\right)-p\left(\phi_{\sigma(j)}\right)+1=\lambda_{i}-\mu_{j}-i+j$, which is the $(i, j)$-entry of the Jacobi-Trudi decomposition matrix for $D$. Therefore, by Lemma 3.25, det $H^{\prime}=$ $s_{D}{ }^{\circ} W s_{E}$, and so

$$
s_{D \circ W}\left(s_{\bar{W}}\right)^{|\widehat{D}|}\left(s_{\bar{O}}\right)^{|\widetilde{D}|}= \pm\left(s_{D} \circ_{W} s_{E}\right),
$$

where the sign on the right-hand side is the same for all $E=W \rightarrow O \uparrow W$.

\section{CONCluding REMARKS}

We wish to conclude by making a remark about Conjecture 3.26 and by introducing two further conjectures.

5.1. Removing Hypothesis V. As noted in Conjecture 3.26, we do not believe that Hypothesis V] (see Hypothesis 3.27) is necessary for Theorem 3.28 to hold. To prove the first assertion of the conjecture, we need to consider skew diagrams $E$ such as

$$
E=\begin{array}{llll} 
& & \times & \\
& \times & \times & w \\
w & \times & & \\
w & \times & &
\end{array} .
$$


Using [2], we can check that Conjecture 3.26 still holds if $D=\underset{\times}{\times} \times$ or if we put $D^{*}$ in place of $D$. Since $s_{D}=s_{D^{*}}$, we conclude that $s_{D^{\circ} W}=s_{D^{*} \circ W E}$, i.e.

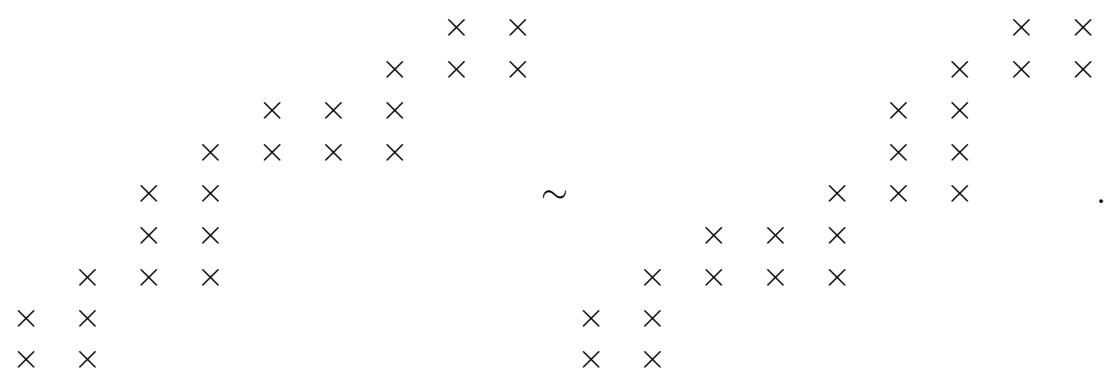

Since $E$ does not satisfy Hypothesis $\mathrm{V}$ this equivalence does not follow from Theorem 3.31, On the other hand, skew-equivalences such as these are explained by Conjecture 3.26. However, since we have been unable to construct outside ribbon decompositions of the skew diagrams in (5.1) that are amenable to our current techniques, it seems that some new ideas will be necessary in order to prove Conjecture 3.26 .

5.2. Skew diagrams equivalent to their transpose. It turns out in practice that there are many skew-equivalences of the form $F \sim F^{t}$. The following result gives an explanation for this.

Proposition 5.1. Suppose $E=W O W$ satisfies Hypotheses [- IV with $E^{t}=E$, $W^{t}=W$ and $W \neq \emptyset$. Then for any skew diagram $D$,

$$
\left(D \circ_{W} E\right)^{t} \sim D \circ_{W} E .
$$

Proof. Since $E$ and $W$ are self-transpose, $E$ must be of the form $W \rightarrow O \uparrow W$ or $W \uparrow O \rightarrow W$. In particular, $E=W O W$ satisfies Hypothesis $\square$ (Hypothesis 3.27) trivially. The result now follows from Lemma 3.19)(iii) and Theorem 3.31

Certainly, if $F=F^{t}$, then $F \sim F^{t}$. We conjecture that the appropriate converse to Proposition 5.1 is also true.

Conjecture 5.2. Suppose a skew diagram $F$ has the property that $F \sim F^{t}$, with $F \neq F^{t}$. Then there exists a skew diagram $E=W O W$ satisfying Hypotheses [ - IV and a skew diagram $D$ such that $F=D \circ_{W} E$ with $E^{t}=E, W^{t}=W$ and $W \neq \emptyset$.

5.3. Necessary and sufficient conditions for skew-equivalence. The strongest result of [1] gives necessary and sufficient conditions for two ribbons to be skew-equivalent. The overarching goal of [14] and the current paper has been to make progress towards extending this result to general skew diagrams. We are now in a position to conjecture such necessary and sufficient conditions.

First, let us state a result that follows by induction from Theorem 3.31

Theorem 5.3. Suppose we have skew diagrams $E_{1}, E_{2}, \ldots, E_{r}$ such that for $i=$ $2, \ldots, r, E_{i}=W_{i} O_{i} W_{i}$ satisfies Hypotheses $\square-\mathbf{V}$. Let $E_{1}^{\prime}$ denote either $E_{1}$ or $E_{1}^{*}$, and for each $i=2, \ldots, r$, let $E_{i}^{\prime}$ and $W_{i}^{\prime}$ denote either $E_{i}$ and $W_{i}$, or $E_{i}^{*}$ and $W_{i}^{*}$. Then

$$
\left(\cdots\left(\left(E_{1} \circ_{W_{2}} E_{2}\right) \circ_{W_{3}} E_{3}\right) \cdots\right) \circ_{W_{r}} E_{r} \sim\left(\cdots\left(\left(E_{1}^{\prime} \circ_{W_{2}^{\prime}} E_{2}^{\prime}\right) \circ_{W_{3}^{\prime}} E_{3}^{\prime}\right) \cdots\right) \circ_{W_{r}^{\prime}} E_{r}^{\prime} \text {. }
$$


Next, let us recall Theorem 4.1 from [1] in our notation, where it was also shown that the $o_{\emptyset}$ operation is associative when applied to ribbons.

Theorem 5.4 ([1, Theorem 4.1]). Two ribbons $\alpha$ and $\beta$ satisfy $\alpha \sim \beta$ if and only if, for some $r$,

$$
\alpha=\alpha_{1} \circ_{\emptyset} \alpha_{2} \circ_{\emptyset} \cdots \circ_{\emptyset} \alpha_{r} \text { and } \beta=\beta_{1} \circ_{\emptyset} \beta_{2} \circ_{\emptyset} \cdots \circ_{\emptyset} \beta_{r},
$$

where, for each $i, \alpha_{i}$ and $\beta_{i}$ are ribbons with either $\beta_{i}=\alpha_{i}$ or $\beta_{i}=\alpha_{i}^{*}$. The skewequivalence class of $\alpha$ will contain $2^{r}$ elements, where $r$ is the number of factors $\alpha_{i}$ in the irreducible factorisation of $\alpha$ such that $\alpha_{i} \neq \alpha_{i}^{*}$.

It transpires that the concept of irreducible factorisation of [1] can be extended to arbitrary skew diagrams.

Definition 5.5. Given a factorisation of a skew diagram $F=D \circ_{W} E$, where $E=W O W$ satisfies Hypotheses [- IV] we say that the factorisation is trivial if the factorisation is any one of the following:

(i) $(1) \circ_{W} F$;

(ii) $F \circ_{\emptyset}(1)$;

(iii) $\emptyset \circ_{F} E$.

We say the factorisation is minimal if it is non-trivial and, among all factorisations of $F, W$ and then $E$ occupies the minimum number of diagonals.

A factorisation $\left(\cdots\left(\left(E_{1} \circ_{W_{2}} E_{2}\right) \circ_{W_{3}} E_{3}\right) \cdots\right) \circ_{W_{r}} E_{r}$ is called irreducible if:

$\circ E_{1}$ only admits trivial factorisations;

○ for $i=2, \ldots, r$ we have $E_{i}=W_{i} O_{i} W_{i}$ satisfies Hypotheses [- IV

○ each factorisation $D_{i} \circ_{W_{i}} E_{i}$ is minimal, where

$$
D_{i}=\left(\cdots\left(\left(E_{1} \circ_{W_{2}} E_{2}\right) \circ_{W_{3}} E_{3}\right) \cdots\right) \circ_{W_{i-1}} E_{i-1} .
$$

Remark 5.6. If $F$ is a ribbon, then we can prove that the irreducible factorisation of $F$ is unique by a proof similar to [1, Theorem 3.6]. Unfortunately, uniqueness does not hold in general, for if $F$ is the left-hand skew diagram of (5.1), then it can be irreducibly factored into

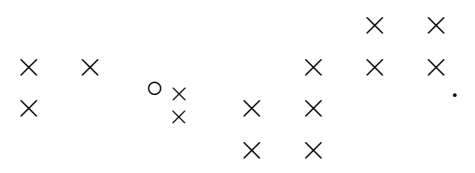

However, since $F=F^{t}$, it can also be factored into

$$
\begin{array}{ccccccc} 
& & & & & \times & \times \\
\times & \times & \circ \times \times & & \times & \times & \times \\
& & \times & & & \\
& & & \times & &
\end{array}
$$

by Lemma 3.19 (iii). Nonetheless, observe that the number of factors $E_{i}$ for which $E_{i} \neq E_{i}^{*}$ is the same for both factorisations.

We now state our main conjecture, of which Theorem 5.4 implies a very special case. 
Conjecture 5.7. Two skew diagrams $E$ and $E^{\prime}$ satisfy $E \sim E^{\prime}$ if and only if, for some $r$,

$$
\begin{aligned}
E & =\left(\cdots\left(\left(E_{1} \circ_{W_{2}} E_{2}\right) \circ_{W_{3}} E_{3}\right) \cdots\right) \circ_{W_{r}} E_{r} \text { and } \\
E^{\prime} & =\left(\cdots\left(\left(E_{1}^{\prime} \circ_{W_{2}^{\prime}} E_{2}^{\prime}\right) \circ_{W_{3}^{\prime}} E_{3}^{\prime}\right) \cdots\right) \circ_{W_{r}^{\prime}} E_{r}^{\prime},
\end{aligned}
$$

where

○ $E_{1}, E_{2}, \ldots, E_{r}$ are skew diagrams;

○ for $i=2, \ldots, r, E_{i}=W_{i} O_{i} W_{i}$ satisfies Hypotheses 【 - IV;

○ $E_{1}^{\prime}=E_{1}$ or $E_{1}^{\prime}=E_{1}^{*}$;

$\circ$ for $i=2, \ldots, r, E_{i}^{\prime}$ and $W_{i}^{\prime}$ denote either $E_{i}$ and $W_{i}$, or $E_{i}^{*}$ and $W_{i}^{*}$.

The skew-equivalence class of $E$ will contain $2^{r}$ elements, where $r$ is the number of factors $E_{i}$ in any irreducible factorisation of $E$ such that $E_{i} \neq E_{i}^{*}$.

Remark 5.8. First, note that it was conjectured [14, Conjecture 9.1] that skewequivalence classes have size a power of 2 , and with our construction we can now predict precisely which power. Secondly, observe that our definition of irreducible factorisation for ribbons differs from that in [1. As an example, the diagram (4) is irreducible under the definition in 1 but is reducible to $(2) \circ_{\emptyset}(2)$ under our definition. However, the powers of 2 in Theorem 5.4 and Conjecture 5.7 are indeed the same. The condition of minimising $W$ first in the irreducible factorisation for skew diagrams ensures that we can naturally pair up terms in the expressions arising from both statements, and those terms that do not pair up will not contribute to the power of 2 .

For an example of a skew-equivalence class of size greater than 4 , we see that in Remark 3.33, $D \circ_{W} E$ is actually equal to $\left(E \circ_{W} E\right) \circ_{W} E$, where $E=(2,1)$ and $W=(1)$. One can check that the 8 skew diagrams of the form $\left(E^{\prime} \circ_{W} E^{\prime \prime}\right){ }^{\circ} E^{\prime \prime \prime}$, with $E^{\prime}, E^{\prime \prime}$ and $E^{\prime \prime \prime}$ each equal to $E$ or $E^{*}$, are all different.

To conclude we present evidence in favour of Conjecture 5.7 Observe that the "if" direction of Conjecture 5.7 would follow from Conjecture 3.26 in the same way that Theorem 5.3 follows from Theorem 3.28 . The only difference is that Hypothesis $\mathrm{V}$ is absent in the conjectures. To support both the converse direction and the skew-equivalence class sizes, we have verified that the conjecture holds for all skew diagrams with at most 20 cells.

\section{ACKNOWLEDGEMENTS}

The authors would like to thank Nantel Bergeron and Vic Reiner for comments that helped to spark productive lines of investigation. The Littlewood-Richardson calculator [2] and the SF package [18] aided invaluably in data generation. We also thank the anonymous referee for thoughtful suggestions that improved the exposition.

\section{REFERENCES}

1. Louis J. Billera, Hugh Thomas, and Stephanie van Willigenburg. Decomposable compositions, symmetric quasisymmetric functions and equality of ribbon Schur functions. Adv. Math., 204(1):204-240, 2006. MR2233132 (2007b:05207)

2. Anders S. Buch. Littlewood-Richardson calculator, 1999. Available from http://www . math. rutgers. edu/ asbuch/lrcalc/. 
3. William Y. C. Chen, Guo-Guang Yan, and Arthur L. B. Yang. Transformations of border strips and Schur function determinants. J. Algebraic Combin., 21(4):379-394, 2005. MR2153932 (2006b:05122)

4. Harm Derksen and Jerzy Weyman. On the Littlewood-Richardson polynomials. J. Algebra, 255(2):247-257, 2002. MR 1935497 (2003i:16021)

5. A. M. Hamel and I. P. Goulden. Planar decompositions of tableaux and Schur function determinants. European J. Combin., 16(5):461-477, 1995. MR.1345693 (96k:05205)

6. Roger A. Horn and Charles R. Johnson. Matrix analysis. Cambridge University Press, Cambridge, 1985. MR832183(87e:15001)

7. R. C. King, C. Tollu, and F. Toumazet. Stretched Littlewood-Richardson and Kostka coefficients. In Symmetry in physics, volume 34 of CRM Proc. Lecture Notes, pages 99-112. Amer. Math. Soc., Providence, RI, 2004. MR.2056979(2005e:05153)

8. Allen Knutson and Terence Tao. The honeycomb model of $\mathrm{GL}_{n}(\mathbf{C})$ tensor products. I. Proof of the saturation conjecture. J. Amer. Math. Soc., 12(4):1055-1090, 1999. MR1671451 (2000c:20066)

9. D.E. Littlewood and A.R. Richardson. Group characters and algebra. Philos. Trans. Roy. Soc. London, Ser. A, 233:99-141, 1934.

10. I. G. Macdonald. Symmetric functions and Hall polynomials. Oxford Mathematical Monographs. The Clarendon Press Oxford University Press, New York, second edition, 1995. With contributions by A. Zelevinsky, Oxford Science Publications. MR1354144 (96h:05207)

11. Hariharan Narayanan. On the complexity of computing Kostka numbers and LittlewoodRichardson coefficients. J. Algebraic Combin., 24(3):347-354, 2006. MR 2260022 (2007g:05187)

12. Kevin Purbhoo. Vanishing and nonvanishing criteria in Schubert calculus. Int. Math. Res. Not., 2006:Article ID 24590, 38 pages, 2006. MR2211140 (2007b:14119)

13. Etienne Rassart. A polynomiality property for Littlewood-Richardson coefficients. J. Combin. Theory Ser. A, 107(2):161-179, 2004. MR2078884(2005d:05149)

14. Victor Reiner, Kristin M. Shaw, and Stephanie van Willigenburg. Coincidences among skew Schur functions. Adv. Math., 216(1):118-152, 2007. MR2353252

15. Bruce E. Sagan. The symmetric group, volume 203 of Graduate Texts in Mathematics. Springer-Verlag, New York, second edition, 2001. Representations, combinatorial algorithms, and symmetric functions. MR.1824028 (2001m:05261)

16. M.-P. Schützenberger. La correspondance de Robinson. In Combinatoire et représentation du groupe symétrique (Actes Table Ronde CNRS, Univ. Louis-Pasteur Strasbourg, Strasbourg, 1976), pages 59-113. Lecture Notes in Math., Vol. 579. Springer, Berlin, 1977. MR0498826 (58:16863)

17. Richard P. Stanley. Enumerative combinatorics. Vol. 2, volume 62 of Cambridge Studies in Advanced Mathematics. Cambridge University Press, Cambridge, 1999. MR1676282 (2000k:05026)

18. John R. Stembridge. SF, posets and coxeter/weyl. Available from http://www.math.lsa.umich.edu/ jrs/maple.html.

19. Glânffrwd P. Thomas. Baxter algebras and Schur functions. Ph.D. thesis, University College of Swansea, 1974.

20. Glânffrwd P. Thomas. On Schensted's construction and the multiplication of Schur functions. Adv. in Math., 30(1):8-32, 1978. MR511739 (81g:05022)

Departamento de Matemática, Instituto Superior Técnico, 1049-001 Lisboa, Portugal Current address: Department of Mathematics, Bucknell University, Lewisburg, Pennsylvania 17837

E-mail address: peter.mcnamara@bucknell.edu

Department of Mathematics, University of British Columbia, Vancouver, British COLUmbia, V6T 1Z2, CANADA

E-mail address: steph@math.ubc.ca 